\title{
Activation of a Protein Kinase Via Asymmetric Allosteric Coupling of Structurally Conserved Signaling Modules
}

Authors: Yuxin $\mathrm{Hao}^{1}$, Jeneffer England ${ }^{1}$, Luca Belluci ${ }^{2}$, Emanuele Paci $^{3}$, H. Courtney Hodges $^{4,5,6,7}$, Susan S. Taylor ${ }^{8,9}$ and Rodrigo A. Maillard ${ }^{1 *}$

\begin{abstract}
Affiliations:
${ }^{1}$ Department of Chemistry, Georgetown University, Washington, DC 20057.

${ }^{2}$ NEST, Istituto Nanoscienze del CNR and Scuola Normale Superiore, Pisa, Italy.

${ }^{3}$ Astbury Centre \& School of Molecular and Cellular Biology, University of Leeds, Leeds, UK.

${ }^{4}$ Department of Molecular and Cellular Biology, Baylor College of Medicine, Houston, TX 77030.

${ }^{5}$ Center for Precision Environmental Health, Baylor College of Medicine, Houston, TX 77030.

${ }^{6}$ Dan L Duncan Comprehensive Cancer Center, Baylor College of Medicine, Houston, TX 77030.

${ }^{7}$ Center for Cancer Epigenetics, The University of Texas MD Anderson Cancer Center, Houston, TX 77030.

${ }^{8}$ Department of Pharmacology, University of California, San Diego, La Jolla, CA 92093.

${ }^{9}$ Department of Chemistry and Biochemistry, University of California, San Diego, La Jolla, CA 92093.

*Correspondence to: rodrigo.maillard@georgetown.edu
\end{abstract}




\begin{abstract}
Cyclic nucleotide binding (CNB) domains are universally conserved signaling modules that regulate the activities of diverse protein functions. Yet, the structural and dynamic features that enable the cyclic nucleotide binding signal to allosterically regulate other functional domains remain unknown. We use force spectroscopy and molecular dynamics to monitor in real time the pathways of signals transduced by cAMP binding in protein kinase A (PKA). Despite being structurally conserved, we find that the response of the folding energy landscape to cAMP is domain-specific, resulting in unique but mutually coordinated regulatory tasks: one CNB domain initiates cAMP binding and cooperativity, while the other triggers inter-domain interactions that lock the active conformation. Moreover, we identify a new cAMP-responsive switch, whose stability and conformation depends on cAMP occupancy. Through mutagenesis and nucleotide analogs we show that this dynamic switch serves as a signaling hub, a previously unidentified role that amplifies the cAMP binding signal during the allosteric activation of PKA.
\end{abstract}




\section{INTRODUCTION}

Throughout evolution, nature has utilized conserved protein domains as regulatory signaling modules $^{1-6}$. In multi-domain assemblies, these signaling modules communicate and transduce ligand binding signals to other functional domains, thereby enabling diverse responses to intracellular signaling cascades ${ }^{7,8}$. Cyclic nucleotide binding $(\mathrm{CNB})$ domains are ubiquitous and structurally conserved signaling modules that regulate the activities of protein kinases, guanine nucleotide exchange factors, ion channels, and transcription factors in response to cyclic nucleotides ${ }^{1}$. To date, a general understanding of how the activity of CNB domains can be adapted to regulate a diverse array of protein functions remains rudimentary.

Here, we used optical tweezers in combination with steered molecular dynamics to study the mechanisms that link ligand binding and inter- domain communication with allosteric regulation of cAMP-dependent protein kinase A (PKA). PKA is an archetype of cyclic nucleotide-dependent protein kinases that is composed of regulatory and catalytic subunits (Fig. 1a) ${ }^{9}$. The phosphorylating activity of the catalytic subunit is allosterically driven by two CNB domains of the regulatory subunit, termed $\mathrm{CNB}-\mathrm{A}$ and $\mathrm{CNB}-\mathrm{B}^{10-12}$. $\mathrm{cAMP}$ binding starts in the CNB-B domain and enables binding of a second cAMP molecule to the CNB-A domain, resulting in a profound conformational change that unleashes the activity of catalytic subunits ${ }^{10}$.

Our studies show that cAMP binding to the two CNB domains of PKA propagates a reorganization of inter-domain contact nodes that reshape the folding energy landscape of the protein. Changes in the energy landscape are unique to each CNB domain and arise from both ligand-binding and inter-domain interactions. We identify a division of labor among CNB domains: the CNB-B domain is responsible for initiating and triggering cAMP binding cooperativity while the CNB-A domain induces strong inter-domain interactions that lock the 
entire protein complex into its active conformation. Moreover, we identify a new cAMPresponsive structural element, the $\mathrm{N} 3 \mathrm{~A}$ motif, that switches in stability and conformation depending on cAMP occupancy and inter-domain contacts. Through mutagenesis and the use of cyclic nucleotide analogs, we show that this ligand-responsive switch is selective to cAMP and serves as a signaling hub, amplifying the cAMP binding signal during the allosteric activation of PKA. Altogether, this study illustrates how each structurally conserved CNB domain has evolved to carry out unique but mutually coordinated regulatory tasks in a macromolecular assembly. Our work reveals new operating principles for ligand-directed protein allostery mediated by widely conserved signaling modules.

\section{RESUlts}

Multiple Pulling Geometries with Optical Tweezers Allow the Dissection of Folding Energy

\section{Landscape Parameters}

To study the CNB domain communication mechanisms triggered by cAMP, we began by perturbing the free energy landscape of the PKA regulatory subunit with optical tweezers (Fig. 1b, left). We attached DNA handles via thiol chemistry to two cysteines engineered at specific positions in the protein (see Methods) ${ }^{13,14}$. The handle position determines the direction and region of the protein subjected to the force applied through the optical tweezers (i.e., pulling geometry) ${ }^{15-}$ 17. We generated three PKA regulatory subunit constructs with unique pulling geometries to probe cAMP binding coupled to inter-domain interactions (Fig 1b, right). In type-I constructs, force is applied to the isolated CNB domains to study the effect of cAMP binding on the free energy landscape of each domain. In type-II constructs, force is applied selectively to one CNB domain 
in the presence of the neighboring one. This pulling geometry allows us to directly assess how cAMP binding induces inter-domain interactions, a strategy that would otherwise be inaccessible with bulk methods or single molecule fluorescence techniques. In type-III constructs, force is applied across both CNB domains simultaneously, allowing non-contiguous regions of the protein to respond to force, thereby probing long-range allosteric interactions, either in the presence or absence of cAMP.

We separately tethered each type of protein construct between two polystyrene beads in the optical tweezers (Fig. 1b, left and Methods). By gradually increasing and decreasing the tension across a single protein ("force ramp" pulling), we observed one or more rips in the resulting forceextension curves that correspond to unfolding and refolding events, respectively (Fig. 1c). In the apo state, the isolated $\mathrm{CNB}$ domains unfold at a similar average force, $\mathrm{F}_{\mathrm{avg}} \sim 7-9 \mathrm{pN}$, and with similar unfolding kinetic parameters: the lifetime of the folded state extrapolated to zero force, $\tau_{0, \mathrm{~F}}$, is $1.1-1.6 \cdot 10^{3} \mathrm{~s}$ and the distance to the transition state, $\Delta \mathrm{x}_{\mathrm{F}_{\rightarrow} \mathrm{U}}^{\ddagger}, 4-5 \mathrm{~nm}$ (Fig. 1d and Supplementary Table 1) ${ }^{18,19}$. Analysis of refolding transitions show small differences, wherein the isolated CNBA domain has a shorter unfolded state lifetime, $\tau_{0, \mathrm{U}}$, and a longer $\Delta \mathrm{x}_{\mathrm{U}_{\rightarrow} \mathrm{F}}$ compared to the isolated CNB-B domain (Supplementary Table 1). The selective (type-II constructs) or simultaneous (type-III construct) mechanical manipulation of the CNB domains in the apo state showed indistinguishable unfolding and refolding kinetic parameters compared to their isolated counterparts (Fig. 1e and Supplementary Fig. 1 and 2), indicating that inter-domain interactions within the PKA regulatory subunit are negligible in the absence of $\mathrm{CAMP}^{20}$.

\section{Asymmetric Domain Stabilization Effects Triggered by cAMP Binding}


In contrast to the results obtained in the apo state, the presence of cAMP revealed important differences between the two CNB domains. The unfolding force of the isolated CNB-B domain increases to $\mathrm{F}_{\text {avg }}=12.0 \pm 1.0 \mathrm{pN}$ (Fig. 2a, b, $\mathrm{N}=648$ ), resulting in a $\sim 30$-fold increase of $\tau_{0, \mathrm{~F}}$ (Fig. 2c and Supplementary Table 2). For the isolated CNB-A domain, $\mathrm{F}_{\mathrm{avg}}=17.4 \pm 2.0 \mathrm{pN}$ (Fig. 2d, e, $\mathrm{N}=785)$ and $\tau_{0, \mathrm{~F}}$ increases by a factor of $\sim 7$ (Fig. 2f). The kinetic stabilization conferred by cAMP is also observed during the refolding reaction; both CNB domains had a $\sim$ 4-fold decrease in $\tau_{0, \mathrm{U}}$ (Supplementary Fig. 1). These effects illustrate that the minor structural differences between the two CNB domains (r.m.s.d. $=1.2 \AA$ between $\mathrm{C} \alpha$ atoms) do not reflect the important differences in the folding energy landscape response to cAMP binding.

\section{cAMP Induces Inter-Domain Interactions that Reshape the Folding Energy Landscape}

Having characterized each isolated CNB domain, we studied inter-domain interactions triggered by cAMP using type-II constructs. We find that both CNB domains were stabilized by the presence of their counterpart when bound to the cyclic nucleotide (Fig. 2). Interestingly, the magnitude of stabilization was asymmetric (Supplementary Table 2): The CNB-A domain stabilizes the CNB-B domain by an additional $\sim 8 \mathrm{pN}$, resulting in $\mathrm{F}_{\mathrm{avg}}=19.7 \pm 1.6 \mathrm{pN}(\mathrm{N}=1518)$ and a 4-fold increase in $\tau_{0, \mathrm{~F}}$. The presence of the CNB-B domain induces a mechanical stabilization to the CNB-A domain of $\sim 3 \mathrm{pN}$, resulting in $\mathrm{F}_{\mathrm{avg}}=20.3 \pm 1.4 \mathrm{pN}(\mathrm{N}=1152)$ and a 160 -fold increase in $\tau_{0}$. In the refolding reaction, the presence of the neighboring domain decreases $\tau_{0, \mathrm{U}}$ by 150-fold and 10-fold to the CNB-B and CNB-A domains, respectively (Supplementary Fig. 1). These results show that cAMP binding induces specific but coordinated effects, wherein the CNBB domain stabilizes the folded state of the CNB-A domain, and the CNB-A domain destabilizes the unfolded state of the CNB-B domain. Therefore, the cAMP-dependent communications 
between the CNB domains is bidirectional and asymmetric, highlighting a unique role for each domain in the activation mechanism of PKA.

\section{Identification of a cAMP-Responsive Dynamic Switch}

We hypothesized that changes in contour length upon unfolding $\left(\Delta \mathrm{L}_{\mathrm{c}}\right)$ might also reveal important differences in the native folded structures of the CNB domains upon binding cAMP. While the mechanical unfolding of the CNB-B domain in all three types of constructs had a $\Delta \mathrm{L}_{\mathrm{c}}$ of $\sim 50 \mathrm{~nm}$, corresponding to a fully folded domain (Supplementary Table 2 ), the CNB-A domain displayed a more complex behavior. The isolated CNB-A domain in the apo state had the expected $\Delta \mathrm{L}_{\mathrm{c}}$ of $45 \mathrm{~nm}$ based on the crystal structure ${ }^{10}$. However, the value of $\Delta \mathrm{L}_{\mathrm{c}}$ decreased to $30 \pm 3 \mathrm{~nm}$ in the presence of cAMP, indicating that a region of the domain was destabilized upon ligand binding (Supplementary Table 2).

We sought to identify which region or secondary structures of the CNB-A domain become unstable upon cAMP binding. The structure of the CNB-A domain is composed of a $\beta$-sandwich fold that forms the cAMP binding pocket, and three N-terminal $\alpha$-helices termed N3A motif. ${ }^{10,11}$ The N3A motif contains $\sim 30$ amino acids, which matches the amount of polypeptide that became unstable after cAMP binding. To test whether the N3A motif is destabilized by cAMP binding, we used two distinct type-III constructs, one with DNA handles attached at residue positions flanking both CNB domains entirely (S110C/S376C) and another construct with handles flanking both CNB domains except the N3A motif (D149C/S376C). The two constructs displayed two major unfolding rips corresponding to the $\mathrm{CNB}$ domains, but only the unfolding trajectory of the $\mathrm{S} 110 \mathrm{C} / \mathrm{S} 376 \mathrm{C}$ construct revealed a small, reversible transition at $\sim 11 \mathrm{pN}$ with a $\Delta \mathrm{Lc}$ of $13 \mathrm{~nm}$ (Fig. 3a). The lack of such small transition in the D149C/S376C construct, which does not directly 
probe the N3A motif, provides evidence that the secondary structures in the CNB-A domain that become unstable upon cAMP binding correspond to the N3A motif (Supplementary Fig. 3). Steered molecular dynamics (SMD) starting from the X-ray structure corroborate our experimental observations (Methods). The SMD trajectories show that the N3A motif unravels first while the rest of the CNB domains remained stably folded in their original cAMP-bound conformation (Fig. 3b-right, and Supplementary Movie 1). In contrast, SMD trajectories in the absence of cAMP several interdomain interactions were lost (Supplementary Fig. 4), resulting in the detachment of the two CNB domains before any secondary structure unfolds, including the N3A motif (Fig. 3bleft, and Supplementary Movie 2).

\section{Conformational Switching of the N3A Motif Requires Integrity of Inter-Domain Contacts}

In contrast to the results obtained with the isolated cAMP-bound CNB-A domain, the typeIII S110C/S376C construct show that the N3A motif is properly folded in the context of the entire regularly subunit. Moreover, a close inspection of trajectories obtained with the selective manipulation of the cAMP-bound CNB-A domain (type-II construct) revealed a two-step unfolding process instead of a single rip (Fig 2d, red arrow). The additional rip had a $\Delta \mathrm{L}_{\mathrm{c}}$ of $\sim 13$ $\mathrm{nm}$, similar to that of the N3A motif. These observations indicate that the CNB-B domain enables the refolding of the N3A motif in the presence of cAMP.

Because our optical tweezers assay permitted to control the sequence of events in the unfolding reaction, we used the type-III S110C/S376C construct to determine whether the N3A motif can refold while the CNB-B domain remains in the unfolded state (Fig. 4a). In this experiment, we applied a force up to $15 \mathrm{pN}$ to unfold both the N3A motif and the CNB-B domain, but not the CNB-A domain. The force was then decreased to $5 \mathrm{pN}$ to maintain the CNB-B domain 
in the unfolded state (refolding transitions begin at forces $<2 \mathrm{pN}$ ). After ten or more pulling and relaxation cycles between $5-15 \mathrm{pN}$, we did not observe any small, reversible transitions at $\sim 11$ $\mathrm{pN}$, which would have corresponded to a folded N3A motif while the CNB-B domain remained unfolded. Thus, we find that the CNB-B domain is strictly required for the N3A motif to refold. This result is in agreement with the structure of the cAMP-bound regulatory subunit that shows the N3A motif docks into a cleft formed between the CNB domains, ${ }^{11}$ establishing several surface

contacts not only with the CNB-A domain and the $\mathrm{BC}$ helix, ${ }^{21}$ but also with the CNB-B domain (Fig. 4b).

Intermediate cAMP-Bound States Reveal Stepwise Stabilization Mechanism Between CNB Domains

Since cAMP binds to PKA in a sequential fashion ${ }^{10}$, thereby populating intermediate-cAMPbound species, we investigated the coupling between the folding status of the N3A motif and interdomain interactions in conditions where only one CNB domain is bound to cAMP. To obtain forceextension curves of intermediate cAMP-bound states, we used the type-III construct S110C/S376C and titrated cAMP between 1-150 nM (Fig. 5a). We find that the CNB-B domain bound to cAMP increases both $\mathrm{F}_{\mathrm{avg}}$ by $\sim 1 \mathrm{pN}$ (KS Test, $p \cong 0$ ) and $\tau_{0, \mathrm{~F}}$ by 2 -fold to the cAMP-free CNB-A domain (Fig. 5b, top). The CNB-A domain bound to cAMP induces a larger stabilization to the cAMPfree $\mathrm{CNB}-\mathrm{B}$ domain, increasing $\mathrm{F}_{\mathrm{avg}}$ by $\sim 3 \mathrm{pN}$ and $\tau_{0, \mathrm{~F}}$ by 3 -fold (Fig. 5b, bottom). These results show that cAMP binding to one $\mathrm{CNB}$ domain is sufficient to initiate stabilizing inter-domain interactions with the neighboring apo CNB domain, but compared to the fully cAMP-bound state these interactions are partial in magnitude (Supplementary Table 3). Moreover, we find that these partial inter-domain interactions are insufficient to drive the folding of the N3A motif between the 
two CNB domains, i.e., analysis of $\Delta \mathrm{L}_{\mathrm{c}}$ using the WLC model shows that the cAMP-bound CNBA domain interacting with the cAMP-free CNB-B domain does not have a folded N3A motif (Fig. 5c, top). A similar analysis revealed that cAMP binding to the CNB-B domain does not elicit unfolding of the N3A motif in the cAMP-free CNB-A domain (Fig. 5c, bottom). These results strongly support our previous observations showing that unfolding of the N3A motif is solely coupled to cAMP binding to the CNB-A domain, and that the following refolding step of the N3A motif requires the presence of the cAMP-bound CNB-B domain (Fig. 4a).

We also find that partial inter-domain interactions initiated by on-pathway intermediate cAMP-bound states have important functional consequences in terms of cAMP binding affinities and cooperativity. By directly counting unfolding trajectories corresponding to the apo, intermediate and fully bound species as a function of cAMP concentration (Supplementary Fig. 5), we built a single-molecule titration curve, globally fitted the equations for each population species, and determine the microscopic binding constants and cooperativity parameter (Fig. 5d and Supplementary Info). For the first cAMP molecule, the CNB-B domain has a dissociation constant $\left(\mathrm{K}_{\mathrm{d}, \mathrm{CNB}-\mathrm{B}}\right)$ of $10 \pm 1 \mathrm{nM}$ and the $\mathrm{CNB}-\mathrm{A}$ domain has a $\mathrm{K}_{\mathrm{d}, \mathrm{CNB}-\mathrm{A}}=17 \pm 1 \mathrm{nM}$. The affinity for the second cAMP molecule for either CNB domain increases $\sim 3$-fold, indicating positive binding cooperativity. Importantly, the $\mathrm{K}_{\mathrm{d}}$ values of the CNB domains are 3 and 5 times lower than those corresponding to the isolated domains (see Supplementary Info), indicating that as part of the regulatory subunit the CNB domains bind cAMP more tightly (Fig. 5e).

\section{Conformational Stability and Dynamics of the N3A Motif Are Critical During the Allosteric Activation of PKA}


Our results portray the $\mathrm{N} 3 \mathrm{~A}$ motif as a ligand-responsive molecular switch that toggles between different conformations depending on cAMP occupancy and specific domain contacts. This unique character led us to hypothesize that the N3A motif is a critical structural element that mediates cAMP-dependent cooperative interactions between the CNB domains. We tested this hypothesis by placing the mutation R241A in the B/C helix that connects both $\mathrm{CNB}$ domains. In the wild type structure bound to cAMP, R241 interacts with D267 in the CNB-B domain and E200 in the CNB-A domain, thereby bringing the two CNB domains into close proximity for the N3A motif to dock (Fig. 6a, left $)^{11,22,23}$. In the absence of cAMP, unfolding trajectories of R241A using a type-III construct (S110C/S376C) show indistinguishable unfolding parameters compared to wild type (Supplementary Fig. 6). In the presence of cAMP, however, the trajectories of R241A revealed an unfolding pathway that looked similar to that of wild type (Fig. 6a, right and Supplementary Fig. 6), despite some important quantitative differences. Specifically, the average unfolding force for the CNB-B domain in the mutant protein was $\sim 2.5 \mathrm{pN}$ lower compared to wild type, which results in a 3 -fold reduction of $\tau_{0, \mathrm{~F}}(\mathbf{F i g}$. $\mathbf{6 b})$. These values are indistinguishable from those obtained with the isolated CNB-B domain bound to cAMP (Supplementary Table 4), indicating that R241A largely eliminates inter-domain interactions initiated by the cyclic nucleotide. SMD trajectories also show that the first event in the unfolding pathway of R241A is the detachment of the cAMP-bound CNB domains, instead of the unraveling of the N3A motif (Fig. 6c, Supplementary Fig. 4 and Supplementary Movie 3).

To further dissect the role of folding dynamics and conformation of the N3A motif in interdomain interactions, we conducted "force-clamp" experiments, wherein the protein was held at varying constant forces between 10-12 $\mathrm{pN}$, and changes in extension due to unfolding and refolding of the N3A motif were monitored as a function of time (Fig. 6d). Analysis of these 
trajectories using a two-state Bayesian Hidden Markov model (BHMM) ${ }^{24,25}$ revealed that the folded-state lifetime was $\sim 2$ times longer and the unfolded-state lifetime was $\sim 2$-fold shorter for R241A, indicating that the N3A motif in the mutant protein is less dynamic than in wild type (Fig. 6e). In addition, the accompanying $\Delta \mathrm{L}_{\mathrm{c}}$ between the folded and unfolded states for R241A was 6.5 $\pm 1.1 \mathrm{~nm}$ and for wild type was $9.5 \pm 0.5 \mathrm{~nm}$. The difference in folding dynamics and $\Delta \mathrm{L}_{\mathrm{c}}$ indicates that the N3A motif in R241A is stably folded. But because the mutation eliminates cAMPdependent inter-domain interactions (Fig. 6b), it is likely that the folded N3A motif is not docked between the two CNB domains (Fig. 6f), thereby impeding the PKA regulatory subunit to attain its final cAMP-bound conformation. Thus, our single-molecule studies show that R241A mutant imparts cAMP-dependent functional deficiencies ${ }^{26}$ due to a disruption of the conformational dynamics of the N3A motif and its ability to serve as an efficient cAMP-responsive molecular switch.

\section{Dynamic Switching of the N3A Motif is Selective to cAMP}

To study the contribution of the N3A motif conformational switching mechanism towards cyclic nucleotide selectivity, we mechanically manipulated the CNB domains individually (type-I constructs) or simultaneously (type-III construct) in the presence of cGMP. Both CNB domains bound to cGMP show unfolding parameters $\left(\mathrm{F}_{\mathrm{avg}}, \tau_{0, \mathrm{~F}}\right.$, and $\left.\Delta \mathrm{x}_{\mathrm{F}_{\rightarrow} \mathrm{U}}\right)$ that lie in between the values obtained with and without cAMP, indicating partial intra- and inter-domain stabilization effects (Fig. 7a, b and Supplementary Table 5). Interestingly, the isolated cGMP-bound CNB-A domain had a greater $\Delta \mathrm{L}_{\mathrm{c}}$ than its cAMP-bound counterpart (37 nm and $30 \mathrm{~nm}$, respectively), indicating that the N3A motif is not negatively coupled to cGMP, but instead unfolds as a single cooperative unit together with the rest of the domain. In agreement with this interpretation, the force-extension 
curves using a type-III construct with cGMP do not show the small, reversible transition characteristic of the N3A motif (Fig. 7a). Rather, the trajectory revealed two unfolding rips with $\Delta \mathrm{L}_{\mathrm{c}}$ values that reflect the mechanical denaturation of the full-length protein (Fig. 7c and Supplementary Fig. 7). These results provide direct experimental evidence that nucleotide selectivity not only involves previously described defects in binding affinity and cooperativity ${ }^{27}$, but also an attenuation of inter-domain interactions and decoupling of cyclic nucleotide binding from the conformational switching of the N3A motif.

\section{Discussion}

The uncovered intra- and inter-domain communication network that is triggered by cAMP binding cannot be easily inferred from the crystal structure ${ }^{12}$. The network of interactions in PKA involves bidirectional communication that is asymmetric in magnitude, and includes both positive (stabilizing) and negative (destabilizing) coupling interactions that are fine-tuned to attain the final cAMP-bound conformation. Positive coupling promotes stabilizing interfacial interactions between CNB domains. cAMP binding stabilizes the CNB-B domain from $7.6 \mathrm{kcal} / \mathrm{mol}$ to 10.4 $\mathrm{kcal} / \mathrm{mol}$, and the presence of the neighboring cAMP-bound CNB-A domain provides another 3.2 $\mathrm{kcal} / \mathrm{mol}$. We estimate that cAMP binding stabilizes the CNB-A domain from $9.4 \mathrm{kcal} / \mathrm{mol}$ to 11.5 $\mathrm{kcal} / \mathrm{mol}$, and inter-domain interactions confer an additional $4.4 \mathrm{kcal} / \mathrm{mol}$ (Fig. 8a and Supplementary Table 1,2). Negative coupling triggered by cAMP binding effectively melts interactions established between the N3A motif and the catalytic subunit, thereby facilitating the dissociation of the PKA complex. Lastly, the N3A motif folds back between the cAMP-bound CNB domains with a stability of $5.5 \mathrm{kcal} / \mathrm{mol}$. Within this interaction network, we identified the unique routes of domain communication that are disrupted either by R241A or cGMP (Fig. 8b). 
Because the N3A motif is found in many other cyclic nucleotide binding proteins ${ }^{28}$, the uncovered conformational switching mechanism may be a widespread strategy not only to ensure the completion of the allosteric activation process but also to provide ligand selectivity.

In conclusion, our studies reveal a novel allosteric regulation mechanism, in which co-existing positive and negative coupling interactions initiated by cAMP binding are coordinated to sequentially dissociate the PKA complex, commencing from the CNB-B domain, which binds cAMP first and enables binding of the cyclic nucleotide to the CNB-A domain ${ }^{10}$, and finishing with the conformational switch of the N3A motif. Like unzipping "Velcro", this mechanism efficiently peels off the two PKA subunits, where the strong inter-subunit interaction is the result of several smaller interactions that can be broken sequentially, hence the dissociation of the two subunits does not require the crossing of a large free energy barrier but of many small ones. We anticipate similar mechanisms in other protein kinases with catalytic subunits that require the dissociation of regulatory signaling modules ${ }^{29}$. Moreover, the allosteric mechanisms we describe here may be amplified in the PKA hetero-tetramer composed of two regulatory and two catalytic subunits, where the potential cross talk between PKA subunits is expanded. The single-molecule approach exploiting optical tweezers in conjunction of molecular dynamics simulations presented here can be extended to map allosteric effects of disease mutations or inhibitor binding in other kinases or multi-domain assemblies ${ }^{30-34}$. 


\section{ONLINE Methods}

\section{Mutagenesis, Expression and Purification of the PKA Regulatory Subunit and Isolated}

\section{CNB Domains}

The PRSET plasmid harboring the Bos taurus regulatory subunit gene, isoform RI $\alpha$ containing residues 91 to 379 of the full-length sequence was used. To obtain isolated CNB domains, the sequence of the neighboring CNB domain was deleted by site-directed mutagenesis (QuikChange II Agilent). The isolated CNB-A domain contains residues 91 to 243. The isolated CNB-B domain contains residues 243 to 379 . The mutations C345A and C360A were introduced in the CNB-B domain to prevent undesired reactions with the thiol-modified dsDNA handles. To manipulate each individual CNB domain (Type-I constructs) we introduced the mutations S110C/M243C and M243C/S376C for the isolated CNB-A and CNB-B domains, respectively. To manipulate either the CNB-A domain or the CNB-B domain selectively (Type-II constructs) we introduced into the regulatory subunit the mutations S110C/M243C and M243C/S376C, respectively. To manipulate both CNB domains simultaneously (Type-III construct), we introduced into the regulatory subunit the mutations S110C/S376C. All the protein constructs were expressed in BL21(DE3) (NEB) and purified as described previously ${ }^{11,20,35}$. Briefly, the protein was expressed in BL21(DE3) competent cells overnight at $18^{\circ} \mathrm{C}$ with $1 \mathrm{mM}$ IPTG. The cells were in $20 \mathrm{mM}$ MES, $100 \mathrm{mM}$ NaCl, 2 mM EGTA, 2 mM EDTA, 5 mM DTT, pH 6.5 and the spin supernatant was precipitated with $40 \%$ ammonium sulfate before binding to a cAMP-coupled agarose resin. The protein was eluted from the resin with cGMP and run on a size exclusion column to remove the excess cGMP.

\section{Attachment of dsDNA Handles to Protein Constructs}

We followed a protocol previously published by the Maillard laboratory ${ }^{14}$. Briefly, the purified target protein was concentrated to $\sim 5 \mathrm{mg} / \mathrm{mL}$ in $10 \mathrm{mM}$ DTT to reduce all cysteine residues. The 
protein solution was run through 3 Micro Bio-Spin columns (Bio-Gel P6, Biorad) to remove the DTT before adding $10 \mathrm{mM}$ 2,2'-dithiodipyriine (DTDP, Sigma) for 2 hours at room temperature. The unreacted DTDP was removed from the modified protein using 3 additional Micro Bio-Spin columns. The DTDP-activated protein and two different 30-bp 5'-Thiol-modified dsDNA oligos were combined in a 1-to-1 molar ratio and incubated overnight at $4^{\circ} \mathrm{C}$. The resulting protein-oligo chimera was stored at $-80^{\circ}$ C. Each $30-b p$ 5'-Thiol-modified dsDNA oligo used in the formation of the protein-oligo chimera has a unique non-palindromic overhang that is used to ligate 350-bp dsDNA handles modified with either biotin or digoxigenin in their 5' end. The cross-linking reaction was done in the DNA crosslinking buffer (50 mM Tris, $100 \mathrm{mM} \mathrm{NaCl}, \mathrm{pH}$ 7.6). Single molecule optical tweezers measurements of protein constructs in the apo state were obtained by gradient elution (using cAMP) of the protein-oligo chimera from a cAMP-coupled agarose resin. The first eluted samples have an initial cAMP concentration of $20 \mu \mathrm{M}$, which is further diluted to a final concentration of $\sim 0.02 \mathrm{nM}\left(100 \text {-fold }<K_{\mathrm{d}}\right)^{36}$ before using it in the optical tweezers experiment.

\section{Optical Tweezers Measurements}

All data was collected in a MiniTweezers instrument ${ }^{37}$. Measurements were carried out in DNA crosslinking buffer. The protein sample with dsDNA handles was mixed with of $3.1 \mu \mathrm{m}$ polystyrene beads (Spherotech) coated with anti-digoxigenin antibodies (termed AD bead) for 5 min at room temperature. The sample is diluted to $1 \mathrm{~mL}$ before applying it to the optical tweezers microchamber. The optical tweezer experiments were performed in DNA crosslinking buffer in a temperature-controlled room at $20{ }^{\circ} \mathrm{C}$. A $2.1 \mu \mathrm{m}$ bead coated with streptavidin (termed SA bead) is trapped on the tip of a micropipette, whereas the AD bead conjugated with the sample is trapped in the optical trap. To form a single tether, the AD bead in the optical trap was moved towards the 
SA beads on the micropipette tip. A single tether is confirmed by observing overstretching of the DNA handles at $\sim 65 \mathrm{pN}^{38}$. Data was collected in two modes:

a) Force ramp experiments: To mechanically manipulate the target protein construct, we moved the AD bead in the optical trap away and towards the SA bead on the micropipette tip, which results in force-extension curves. The experiment was conducted at a constant pulling velocity of $75 \mathrm{~nm} / \mathrm{s}$, with a 10 second refolding time at $2 \mathrm{pN}$. Data was collected at a sampling rate of $200 \mathrm{~Hz}$. Rupture forces representing the cooperative unfolding of one or more protein domains and their associated extension changes were analyzed using a custom-built program implemented in MATLAB software. Unfolding force probability distributions obtained from force-extension curves were transformed to folded state lifetimes as a function of force and analyzed using the methodology described by Dudko et $a l^{18,19}$. (Full details are provided in the Supplementary Information)

b) Force clamp experiments: the protein was stretched and then held at a desired constant force using constant-force feedback loop. Changes in position at a particular force were recorded at a frequency of $500 \mathrm{~Hz}$ for the R241 A mutant and $1000 \mathrm{~Hz}$ for the wild type protein. The data from force clamp experiments was analyzed using a Bayesian Hidden Markov Model (BHMM) approach ${ }^{24,39}$.

\section{Molecular Dynamics (MD) and Steered Molecular Dynamic (SMD) Simulation}

MD and SMD simulation were performed with NAMD (v2.12) ${ }^{40}$. The CHARMM27 force field $^{41}$ was used for the protein and counterions and the TIP3P ${ }^{42}$ for water. Parameters for cyclic adenosine monophosphate (cAMP) were obtained with the CHARMM general force field $(\mathrm{CGenFF})^{43}$. The holo state was modeled starting from the X-ray structure (PDB code: 1RGS) ${ }^{11}$. The apo state was obtained by removing cAMP from both the binding sites. Regulatory subunit of 
protein kinase A (PKA) with and without cAMP were solvated in a cubic box of $90 \AA$ side. Systems included 24,200 water molecules and counterions were added to guarantee charge neutrality. The bonds between hydrogens and heavy atoms were constrained with the SHAKE algorithm ${ }^{44}$. The rRESPA multiple time step method ${ }^{45}$ was employed where long-range electrostatic interactions are updated every $4 \mathrm{fs}$, and all the other interactions every 2 fs. Periodic boundary conditions (PBC) were used and the long-range electrostatics was treated with the particle-mesh-Ewald (PME) method $^{46}$ using a grid of $81 \times 81 \times 81$. The cut off for non-bonded interactions was set to $10 \AA$, and the switching function was applied to smoot interactions between 9 and $10 \AA$. Simulations were conducted in the NPT ensemble. Temperature was set to $310 \mathrm{~K}$ through a Langevin thermostat ${ }^{47}$ and pressure was set to 1 atm through a isotropic Langevin piston manostat ${ }^{48}$. The systems were first minimized (2000 steps of conjugate gradient) and equilibrated for 800 ps with the atoms of protein restrained to their initial positions. Production runs for both systems where $230 \mathrm{~ns}$ long. The holo structure at $90 \mathrm{~ns}$ was used to model the R241A mutant. The R241A mutant with cAMP was minimized and equilibrated as above and production runs was $200 \mathrm{~ns}$ long.

Root mean square deviation (RMSD) was evaluated over the $\mathrm{C} \alpha$ atoms considering all residues between 119 and 370, excluding the six residues of the unstructured N-and C-terminal tails. RMSD based clustering over $\mathrm{C} \alpha$ atoms was performed with Wordom $^{49}$ in the last $50 \mathrm{~ns}$ of simulation using a threshold of $2 \AA$ (Supplementary Fig. 4).

$\mathrm{SMD}^{50}$ simulations were performed starting from a set of conformations from the production phase. For each selected conformation, the protein was centered into the box and rotated to place the $\mathrm{C} \alpha$ atoms of the first and the last residue along the $x$-axis. To take in account the elongation of the protein during the pulling, the box was enlarged, by adding water molecules along the $x$ direction, by $170 \AA$. Finally, the box for SMDs was $260 \times 90 \times 90 \AA^{3}$ and the grid for the PME set 
to $256 \times 81 \times 81$. The solvent was equilibrated for 400 ps harmonically restraining the protein in its original conformation and another 400 ps during which the restraints were progressively turned off. All SMDs were conducted in the NPT ensemble by using the same parameters employed to carried out the MD simulations. The final conformations were then used as starting point for the SMD simulations. The SMDs were performed by restraining the $\mathrm{C} \alpha$ atom of the first residue to its initial coordinates and applying the pulling constant velocity to a dummy atom attached via a virtual spring to the $\mathrm{C} \alpha$ atom of last residue: the spring constant was set to $0.5 \mathrm{kcal} \mathrm{mol}^{-1} \AA^{-2}$ and the pulling velocity was set to $2.5 \AA$ /ns along the $x$ direction (Supplementary Fig. 4). 


\section{REFERENCES}

1. Kannan, N. et al. Evolution of allostery in the cyclic nucleotide binding module. Genome Biol. 8, (2007).

2. Berman, H. M. et al. The cAMP binding domain: an ancient signaling module. Proc. Natl. Acad. Sci. U. S. A. 102, 45-50 (2005).

3. Minton, K. Cell signalling: Dual specificity of SH2 domains. Nature Reviews Molecular Cell Biology 17, 266 (2016).

4. Mayer, B. J. SH3 domains: complexity in moderation. J. Cell Sci. 114, 1253-1263 (2001).

5. Pawson, T. Protein modules and signalling networks. Nature 373, 573-580 (1995).

6. Nourry, C., Grant, S. G. N. \& Borg, J. PDZ Domain Proteins : Plug and Play! Sci. Signal. 2003, re7 (2003).

7. Pawson, T. \& Nash, P. Assembly of cell regulatory systems through protein interaction domains. Science (2003).

8. Smock, R. G. \& Gierasch, L. M. Sending signals dynamically. Science (2009).

9. Taylor, S. S., Ilouz, R., Zhang, P. \& Kornev, A. P. Assembly of allosteric macromolecular switches: lessons from PKA. Nat. Rev. Mol. Cell Biol. 13, 646-58 (2012).

10. Kim, C., Cheng, C. Y., Saldanha, S. A. \& Taylor, S. S. PKA-I Holoenzyme Structure Reveals a Mechanism for cAMP-Dependent Activation. Cell 130, 1032-1043 (2007).

11. Su, Y. et al. Regulatory subunit of protein kinase A: structure of deletion mutant with cAMP binding domains. Science 269, 807-813 (1995).

12. Zhang, P. et al. Structure and allostery of the PKA RII $\beta$ tetrameric holoenzyme. Science 335, 712-716 (2012). 
13. Cecconi, C., Shank, E. A., Bustamante, C. \& Marqusee, S. Direct observation of the threestate folding of a single protein molecule. Science 309, 2057-60 (2005).

14. Hao, Y., Canavan, C., Taylor, S. S. \& Maillard, R. A. Integrated Method to Attach DNA Handles and Functionally Select Proteins to Study Folding and Protein-Ligand Interactions with Optical Tweezers. Sci. Rep. 7, (2017).

15. Shank, E. a, Cecconi, C., Dill, J. W., Marqusee, S. \& Bustamante, C. The folding cooperativity of a protein is controlled by its chain topology. Nature 465, 637-40 (2010).

16. Mandal, S. S. et al. Nanomechanics of the substrate binding domain of Hsp70 determine its allosteric ATP-induced conformational change. Proc. Natl. Acad. Sci. (2017).

17. Guinn, E. J., Tian, P., Shin, M., Best, R. B. \& Marqusee, S. A small single-domain protein folds through the same pathway on and off the ribosome. Proc. Natl. Acad. Sci. (2018).

18. Dudko, O. K., Hummer, G. \& Szabo, A. Theory, analysis, and interpretation of singlemolecule force spectroscopy experiments. Proc. Natl. Acad. Sci. 105, 15755-15760 (2008).

19. Dudko, O. K., Hummer, G. \& Szabo, A. Intrinsic rates and activation free energies from single-molecule pulling experiments. Phys. Rev. Lett. 96, (2006).

20. England, J. P. et al. Switching of the folding-energy landscape governs the allosteric activation of protein kinase A. Proc. Natl. Acad. Sci. 115, E7478-E7485 (2018).

21. Vehlow, C. et al. CMView: Interactive contact map visualization and analysis. Bioinformatics 27, 1573-1574 (2011).

22. Ries, J., Kaplan, C., Platonova, E., Eghlidi, H. \& Ewers, H. A simple, versatile method for GFP-based super-resolution microscopy via nanobodies. Nat. Methods 9, 582-584 (2012). 
23. Diller, T. C., Madhusudan, Xuong, N.-H. \& Taylor, S. S. Molecular Basis for Regulatory Subunit Diversity in cAMP-Dependent Protein Kinase: Crystal Structure of the Type II $\beta$ Regulatory Subunit. Structure 9, 73-82 (2001).

24. Robert, C. P., Celeux, G. \& Diebolt, J. Bayesian estimation of hidden Markov chains: a stochastic implementation. Stat. Probab. Lett. 16, 77-83 (1993).

25. Kaiser, C. M., Goldman, D. H., Chodera, J. D., Tinoco, I. \& Bustamante, C. The ribosome modulates nascent protein folding. Science 334, 1723-7 (2011).

26. Barros, E. P. et al. Electrostatic Interactions as Mediators in the Allosteric Activation of Protein Kinase A RIa. Biochemistry 56, 1536-1545 (2017).

27. Shabb, J. B., Buzzeo, B. D., Ng, L. \& Corbin, J. D. Mutating protein kinase cAMPbinding sites into cGMP-binding sites: Mechanism of cGMP selectivity. J. Biol. Chem. 266, 24320-24326 (1991).

28. Akimoto, M. et al. A mechanism for the auto-inhibition of hyperpolarization-activated cyclic nucleotide-gated $(\mathrm{HCN})$ channel opening and its relief by cAMP. J. Biol. Chem. 289, 22205-22220 (2014).

29. Taylor, S. S. \& Kornev, A. P. Protein kinases: Evolution of dynamic regulatory proteins. Trends in Biochemical Sciences 36, 65-77 (2011).

30. Chen, Y. N. P. et al. Allosteric inhibition of SHP2 phosphatase inhibits cancers driven by receptor tyrosine kinases. Nature (2016).

31. Faraldo-Gomez, J. D. \& Roux, B. On the importance of a funneled energy landscape for the assembly and regulation of multidomain Src tyrosine kinases. Proc. Natl. Acad. Sci. (2007). 
32. Lavoie, H. et al. MEK drives BRAF activation through allosteric control of KSR proteins. Nature (2018).

33. Nussinov, R. \& Tsai, C. J. Allostery in disease and in drug discovery. Cell (2013).

34. Thal, D. M., Glukhova, A., Sexton, P. M. \& Christopoulos, A. Structural insights into Gprotein-coupled receptor allostery. Nature (2018).

35. Wu, J., Jones, J. M., Xuong, N. H., Ten Eyck, L. F. \& Taylor, S. S. Crystal structures of RIà subunit of cyclic adenosine 5'-monophosphate (cAMP)-dependent protein kinase complexed with ( R p )-adenosine 3',5'-cyclic monophosphothioate and (S p )-adenosine 3',5'-cyclic monophosphotioate, the phosphothioate analogues of cAM. Biochemistry 43, $6620-6629$ (2004).

36. Lorenz, R. et al. Mutations of PKA cyclic nucleotide-binding domains reveal novel aspects of cyclic nucleotide selectivity. Biochem. J. 474, 2389-2403 (2017).

37. Smith, S. B., Cui, Y. \& Bustamante, C. Optical-trap force transducer that operates by direct measurement of light momentum. Methods Enzymol. 361, 134-162 (2003).

38. Bustamante, C., Marko, J. F., Siggia, E. D. \& Smith, S. Entropic elasticity of lambdaphage DNA. Science 265, 1599-1600 (1994).

39. Kaiser, C. M., Goldman, D. H. \& Chodera, J. D. Nascent Protein Folding. 1723-1728 (2011).

40. Phillips, J. C. et al. Scalable Molecular Dynamics with NAMD. 26, 1781-1802 (2005).

41. MacKerell, A. D. et al. All-Atom Empirical Potential for Molecular Modeling and Dynamics Studies of Proteins ${ }^{\dagger}$. J. Phys. Chem. B 102, 3586-3616 (1998). 
42. Jorgensen, W. L., Chandrasekhar, J., Madura, J. D., Impey, R. W. \& Klein, M. L. Comparison of simple potential functions for simulating liquid water. J. Chem. Phys. 79, 926-935 (1983).

43. Vanommeslaeghe, K. et al. CHARMM general force field: A force field for drug-like molecules compatible with the CHARMM all-atom additive biological force fields. $J$. Comput. Chem. 31, 671-690 (2010).

44. Ryckaert, J.-P., Ciccotti, G. \& Herman, B. J. C. Numerical integration of the cartesian equations of motion of a system with constraints: molecular dynamics of n-alkanes. $J$. Comput. Phys. 23, 327-341 (1977).

45. Tuckerman, M., Berne, B. J. \& Martyna, G. J. Reversible multiple time scale molecular dynamics. J. Chem. Phys. 97, 1990-2001 (1992).

46. Essmann, U. et al. A smooth particle mesh Ewald method. J. Chem. Phys. 103, 85778593 (1995).

47. Grest, G. S. \& Kremer, K. Molecular dynamics simulation for polymers in the presence of a heat bath. Phys. Rev. A 33, 3628-3631 (1986).

48. Feller, S. E., Zhang, Y., Pastor, R. W. \& Brooks, B. R. Constant pressure molecular dynamics simulation: The Langevin piston method. J. Chem. Phys. 103, 4613-4621 (1995).

49. Seeber, M. et al. Software news and updates Wordom: A user-friendly program for the analysis of molecular structures, trajectories, and free energy surfaces. J. Comput. Chem. 32, 1183-1194 (2011).

50. Lu, H. \& Schulten, K. Steered molecular dynamics simulations of force-induced protein domain unfolding. Proteins 35, 453-63 (1999). 
Acknowledgments: We thank Maria Fe Lanfranco and members of the Maillard and Taylor laboratories for constructive discussions on the manuscript. We thank Amy Chau from the Maillard lab for helping in collecting cAMP titration data for the isolated CNB domains. We thank Emília Pécora de Barros from the Amaro lab at UCSD for providing the atomic coordinates of the simulated R241A structure. We thank Joseph Lesniewski from the Jorabchi lab at Georgetown University and Alan Lowe from University College London for assisting in the global fitting code using PyFolding.

Funding: This work was supported by NSF Grant MCB1715572 (R.A.M.), NIH Grants R01GM034921 (S.S.T.), R00CA187565 (H.C.H.), Cancer Prevention \& Research Institute of Texas Grant RR170036 (H.C.H.), the V Foundation grant V2018-003 (H.C.H.), and Gabrielle's Angel Foundation for Cancer Research (H.C.H.).

Author contributions: Y.H. and R.A.M. conceived and designed the research. Y.H. purified and modified all protein constructs. Y.H. and J.P.E performed optical tweezers experiments. L.B. performed the molecular dynamic simulations. Y.H., H.C.H. and R.A.M. analyzed the data. Y.H., E.P., H.C.H., S.S.T. and R.A.M wrote the manuscript.

Competing interests: Authors declare no competing interests.

Data and materials availability: All data is available in the main text or the supplementary materials. 
a

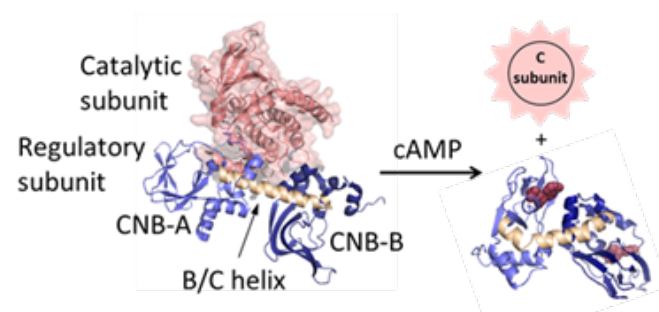

Regulatory subunit

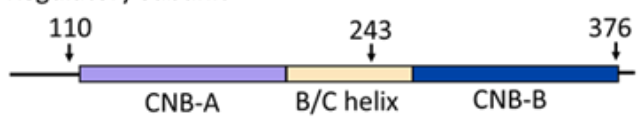

b

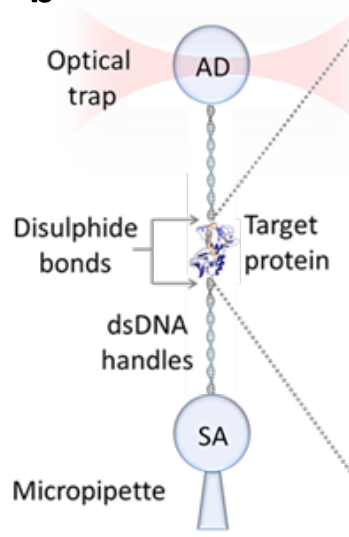

d
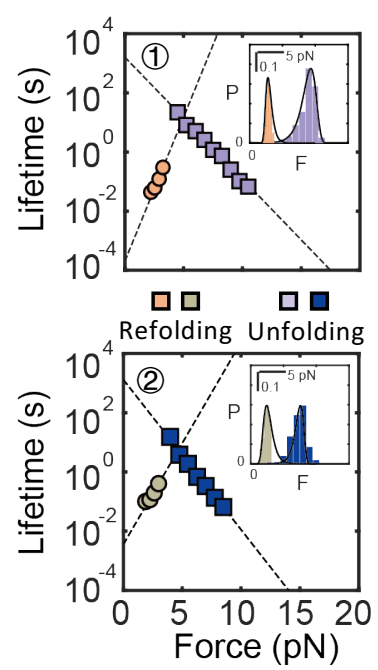

Type-I: Unfolding isolated domains

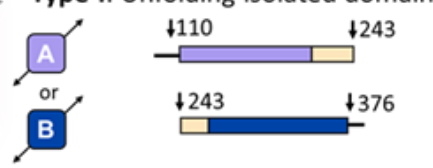

Type-II: Unfolding domains selectively

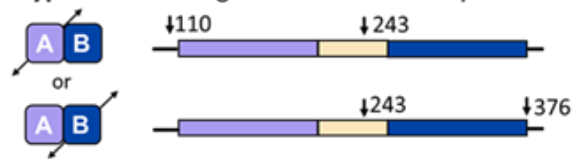

Type-III: Unfolding both domains simultaneously

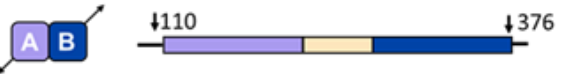

C

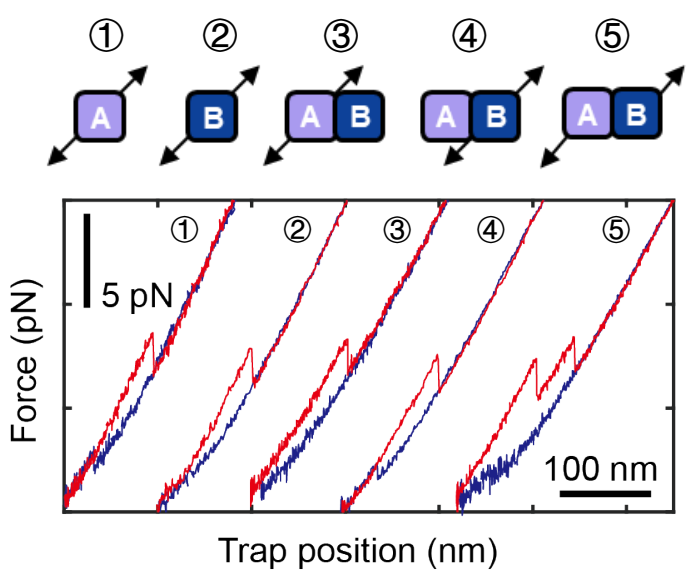

Figure 1 | Experimental design to study allosteric activation in PKA. a, Structure of the inactive PKA holoenzyme (left ${ }^{8}$ and active cAMP-bound regulatory subunit (right) ${ }^{9}$. The arrows on regulatory subunit domain organization indicate the residue positions for DNA handle attachment (bottom, arrows). b, Schematic representation of optical tweezers assay (left) and protein constructs used in this study (right). c, Force-extension curves for all pulling geometries in the apo state (unfolding in red; refolding in blue). d, Force-dependent folded state lifetimes and unfolding force probability distribution (inset) in the apo state. Black lines in insets are the unfolding force distribution reconstructed from force-dependent lifetimes. e, WLC analysis of changes in extension vs. force for the isolated CNB domains (top) and for the $1^{\text {st }}$ and $2^{\text {nd }}$ unfolding rips from the type-III construct (bottom). Dashed lines are the WLC curves for the CNB-A (purple) and CNB-B domains (blue). Numbering in $1 \mathrm{~d}$ and $1 \mathrm{e}$ is the same as in panel $1 \mathrm{c}$. 

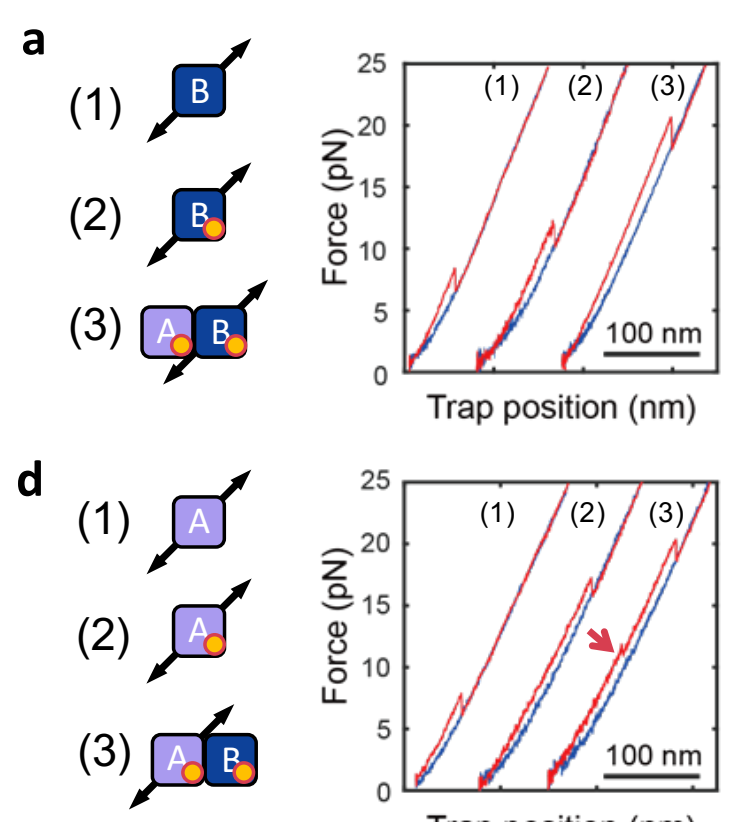

b
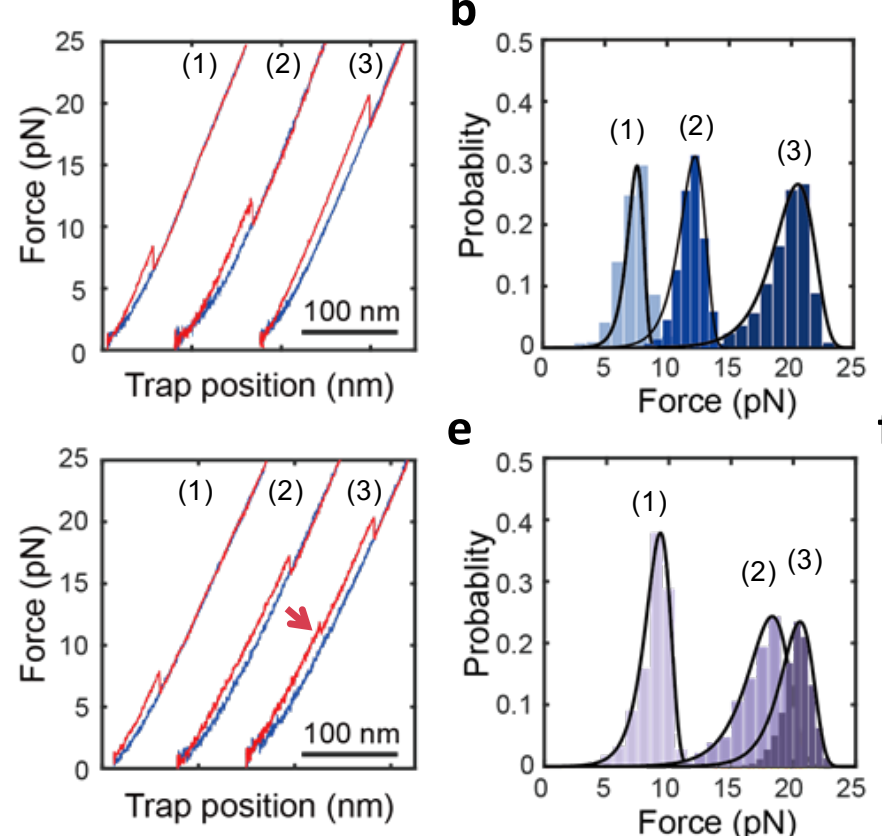

e

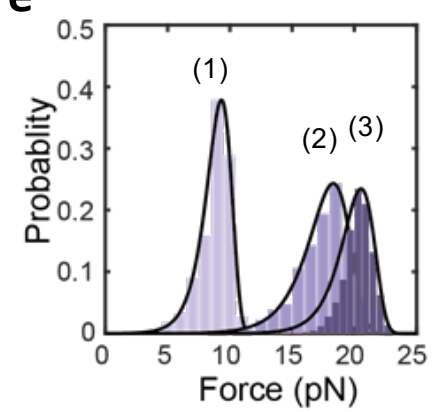

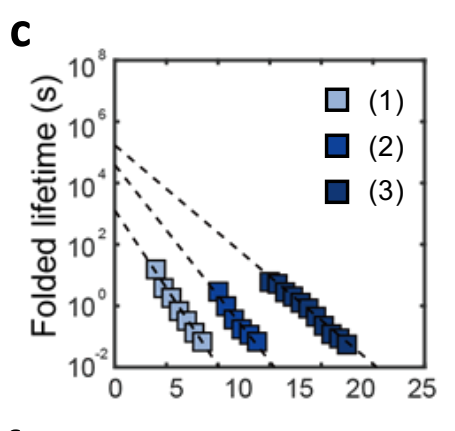

f

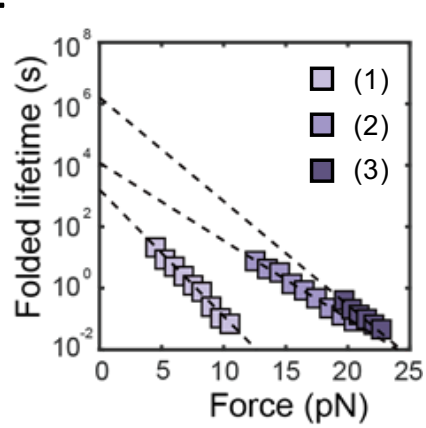

Figure 2 । Selective allosteric effects initiated by cAMP binding. Force-extension curves (a, d), unfolding force probability distributions $(\mathbf{b}, \mathbf{e})$, and force-dependent folded state lifetimes $(\mathbf{c}, \mathbf{f})$ for the CNB-B (top) and CNB-A (bottom) domains. Numbering corresponds to the isolated CNB domains in the apo (1) or cAMP-bound states (2), and selective unfolding of the CNB domains bound to cAMP (3). The red arrow in panel $2 \mathrm{~d}$ indicates the unfolding of the N3A motif. 
a

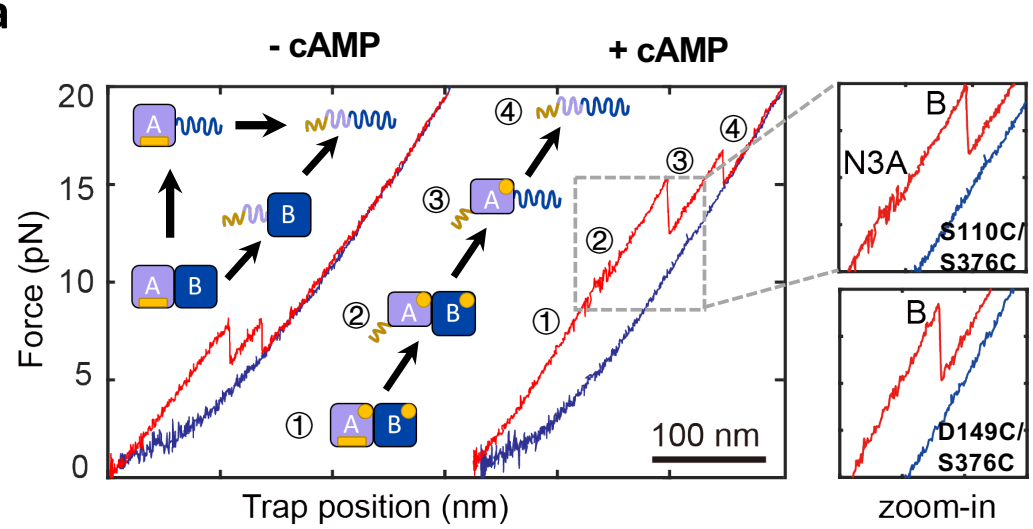

b

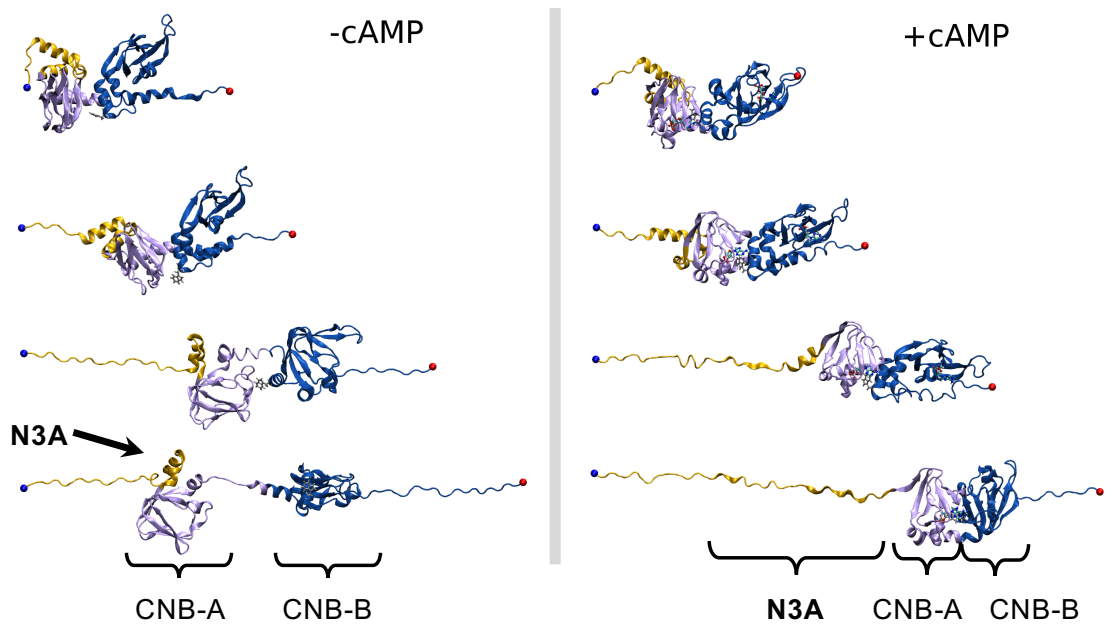

Figure 3 I Dual unfolding pathways of PKA regulatory subunit depending on cAMP occupancy. a, Force-extension curve for the cAMP-free and cAMP-bound regulatory subunit (type-III construct). The cartoon represents the structural transitions occurring during the unfolding trajectory. Zoomed in are the unfolding trajectories of type-III constructs S110C/S376C and D149C/S376C. A detailed analysis of the forces for each unfolding transition refer to Supplementary Information and Supplementary Table 4. b, SMD simulation snapshots of the cAMP-bound (top) and apo (bottom) regulatory subunit. Yellow: N3A motif; Purple: CNB-A; Dark blue: CNB-B domain. 
a

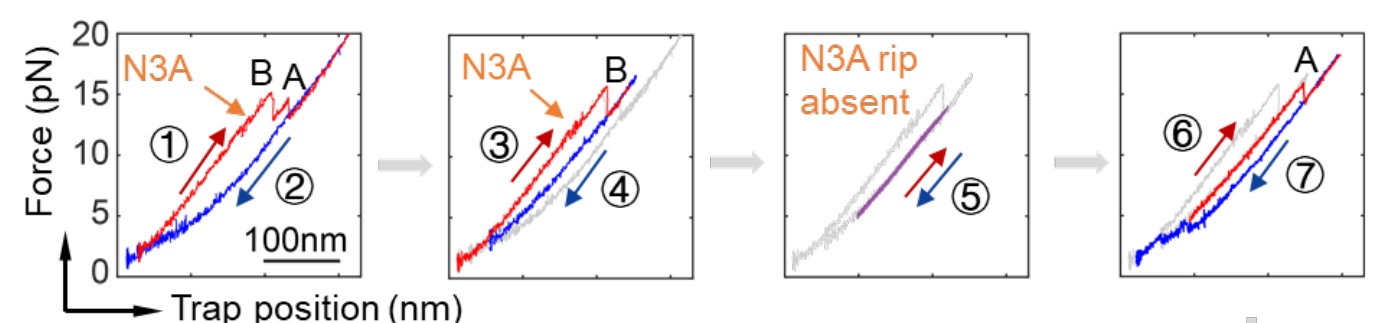

b

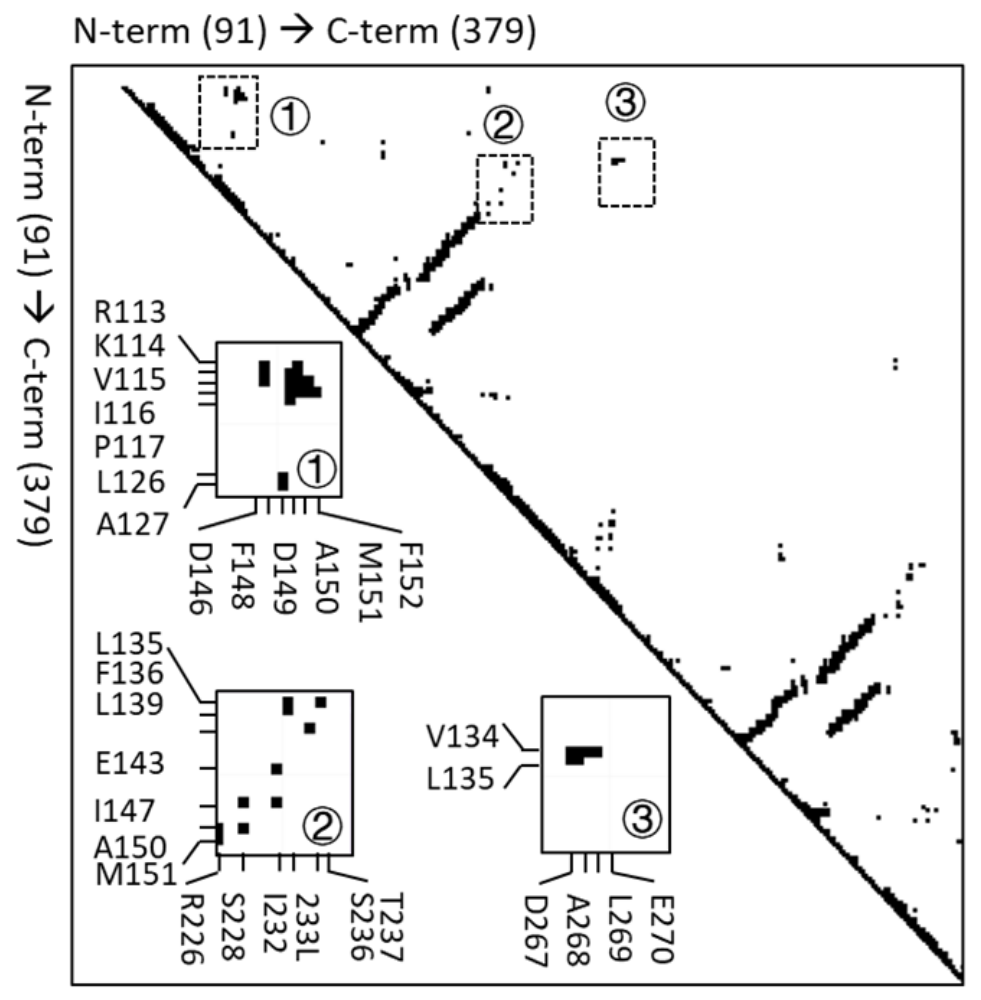

(1) Intra-N3A contacts

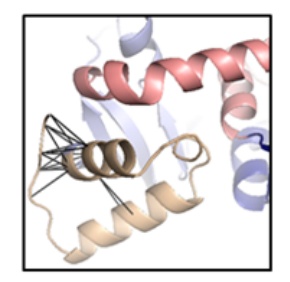

(2) $\mathrm{N} 3 \mathrm{~A}:: \mathrm{B} / \mathrm{C}$ helix contacts

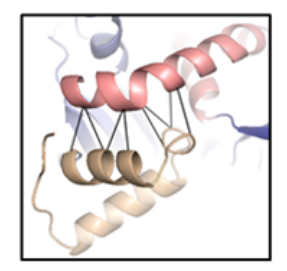

(3) N3A::CNB-B contacts

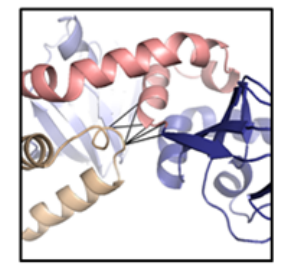

Figure 4 | cAMP-bound CNB-B is required for N3A motif to refold. a, (1) The regulatory subunit unfolds (red) and (2) refolds (blue), revealing the $1^{\text {st }}$ reversible transition corresponding to the N3A motif (orange arrow). (3) In the following cycle, the regulatory subunit was stretched until the N3A motif and the CNB-B domain unfold, while the CNB-A domain remains folded. (4) The force was decreased to $4 \mathrm{pN}$, a force that does not allow the CNB-B domain to refold. (5) The force was then oscillated between $6-15 \mathrm{pN}$ for several cycles $(\sim 20)$ to test whether the N3A motif was able to refold while the CNB-B domains remains unfolded. (6) The force was increased to $20 \mathrm{pN}$ to unfold the CNB-A domain. (7) The force was decreased to $1 \mathrm{pN}$, allowing the complete protein to refold, and begin another set of experiments. The trajectories in gray represent the unfolding pathways from the immediately previous cycle, thereby serving as reference on the progression of experiment. b, Pairwise contact map comparing the interaction established by the N3A motif in the regulatory subunit of PKA (left). The contacts established by the N3A motif were obtained using a $8 \AA$ cutoff (right): (1) contacts established by residues within the N3A motif; (2) contacts between the N3A motif and the B/C helix; and (3) contacts between the N3A motif and the CNB-B domain. Cartoons rendering the three sets of contacts are shown next to the contact map. 
a

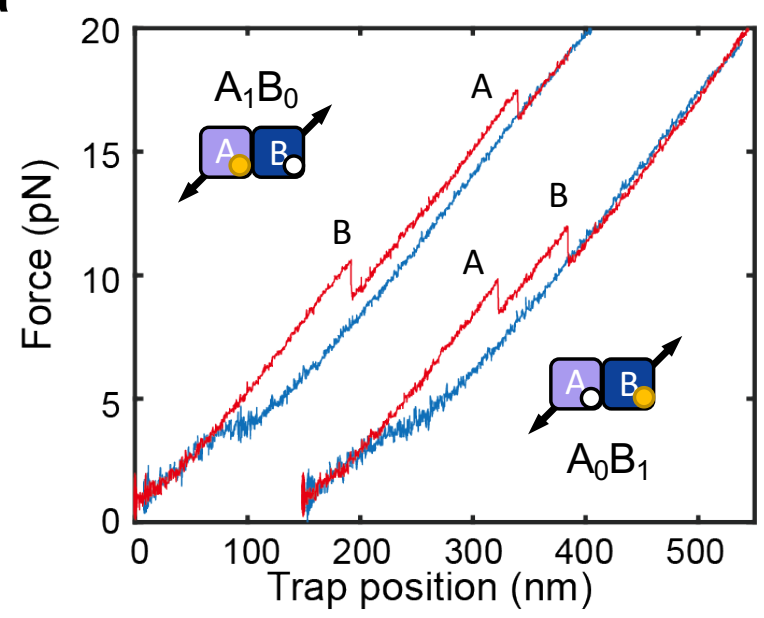

b

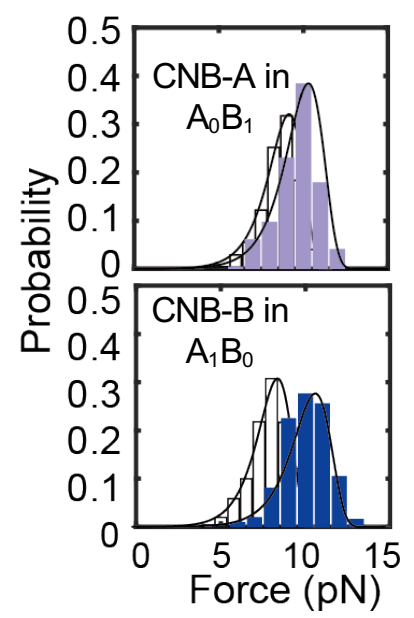

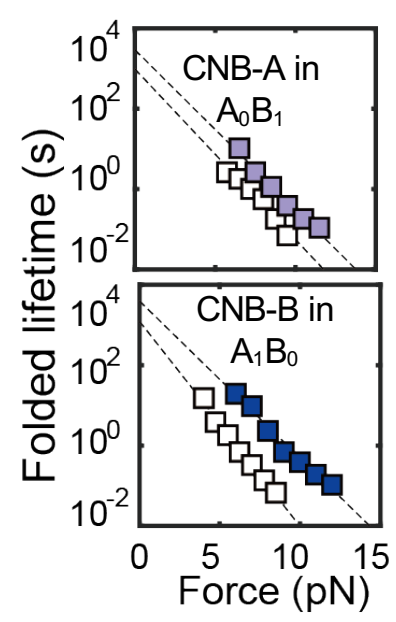
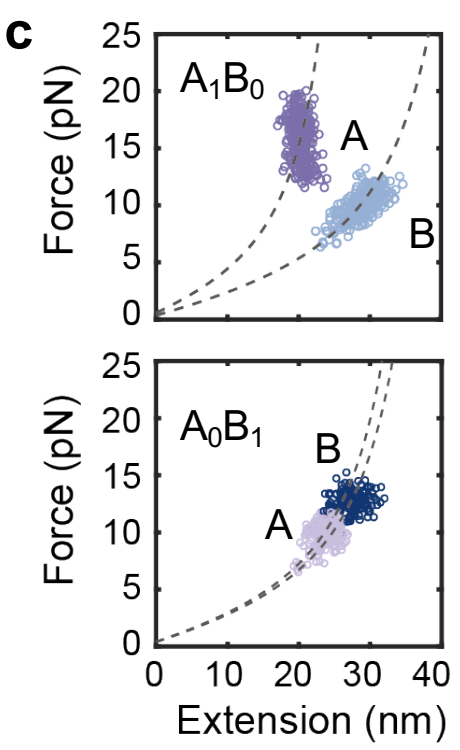
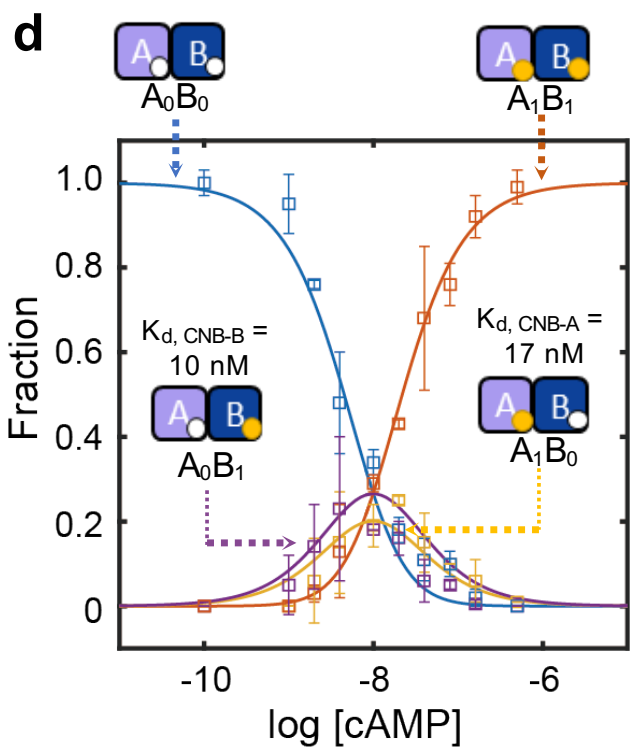
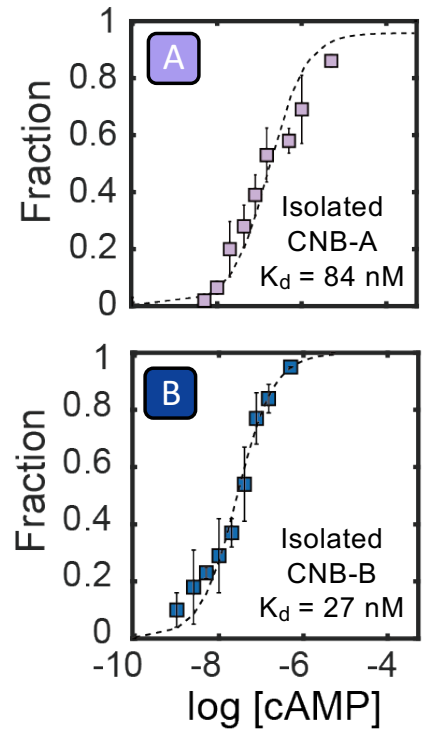

Figure 5 | Stepwise Stabilization of CNB Domains in Partial cAMP-bound States. a, Forceextension curves of intermediate-liganded states $\left(\mathrm{A}_{1} \mathrm{~B}_{0}\right.$ and $\left.\mathrm{A}_{0} \mathrm{~B}_{1}\right)$ for the type-III regulatory subunit S110C/S376C. b, Unfolding force probability distribution and force-dependent folded state lifetimes for intermediate-liganded states: CNB-A domain in $\mathrm{A}_{0} \mathrm{~B}_{1}$ (top) and CNB-B domain in $\mathrm{A}_{1} \mathrm{~B}_{0}$ (bottom). The corresponding isolated domains in the apo state (white bars and symbols) are shown for comparison. Solid lines are the unfolding force distribution reconstructed from force-dependent lifetimes. $\mathbf{c}$, WLC analysis of changes in extension upon unfolding vs. force in $\mathrm{A}_{1} \mathrm{~B}_{0}$ and $\mathrm{A}_{0} \mathrm{~B}_{1}$ for the CNB-A and CNB-B domains (dashed lines). d, cAMP titration plot showing the fraction of apo $\left(\mathrm{A}_{0} \mathrm{~B}_{0}\right)$, intermediate $\left(\mathrm{A}_{1} \mathrm{~B}_{0}, \mathrm{~A}_{0} \mathrm{~B}_{1}\right)$ and fully bound $\left(\mathrm{A}_{1} \mathrm{~B}_{1}\right)$ species. Lines correspond to the global fit to the equations for each species. e, Fractional titration plot of isolated CNB-A (top) and CNB-B (bottom) domains (type-I constructs). The error bar corresponds to the standard deviation of 5-10 different molecules. 
a

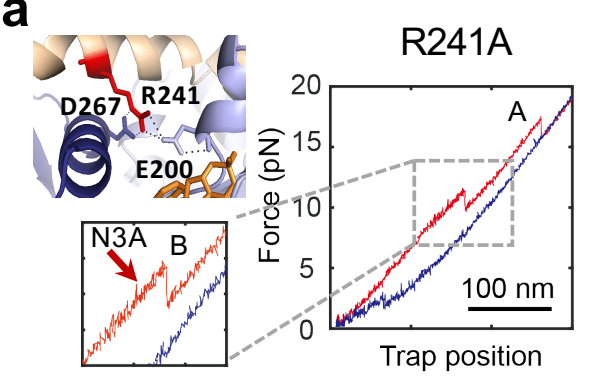

d

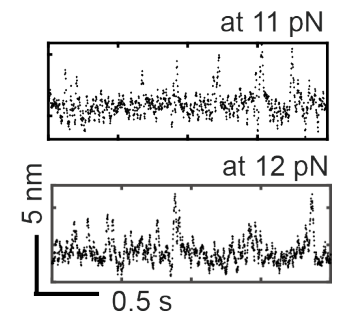

b

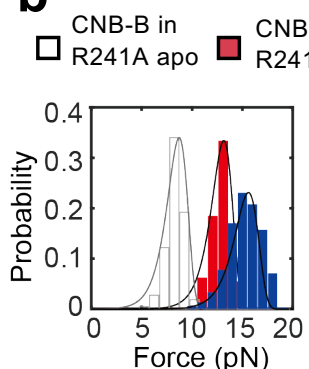

CNB-B in

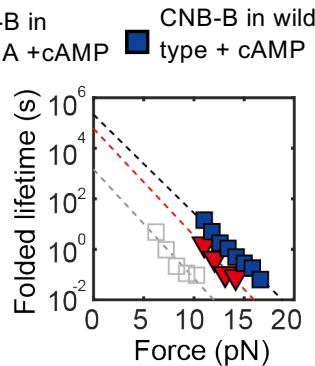

C

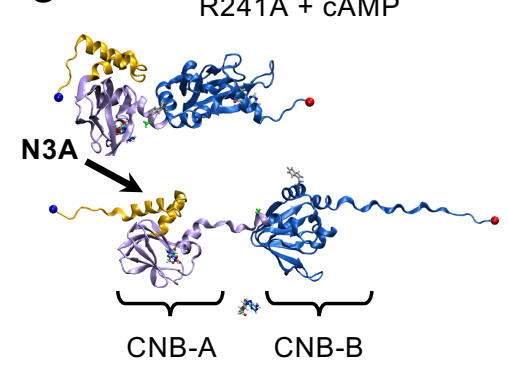

f $\mathrm{R} 241 \mathrm{~A}$

e

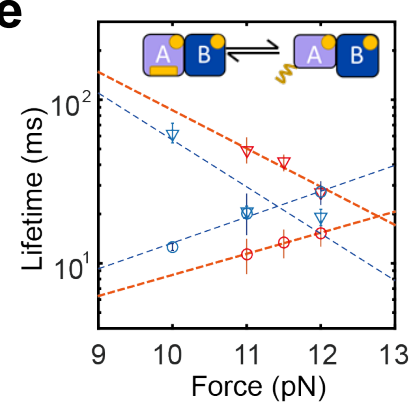

Wild type

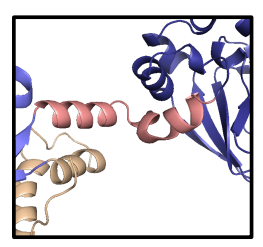

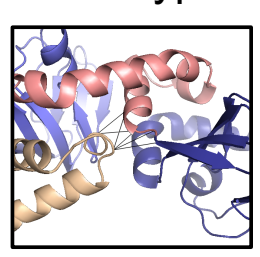

Figure 6 । Perturbation of allosteric networks in PKA by Mutation R241A. a, Residue R241 interacts with both CNB domains through E200 and D267 (PDB 1RGS). Force-extension curve for R241A bound to cAMP (type-III construct S110C/S376C). Zoomed-in is the unfolding rip corresponding to the N3A motif. b, Unfolding probability distributions and force-dependent folded state lifetimes for the CNB-B domain in R241A apo (gray) or bound to cAMP (red). For reference the wild type data was included (blue). Solid lines are the unfolding force distribution reconstructed from force-dependent lifetimes. c, SMD simulation snapshot of the cAMP-bound R241A protein. d, Representative force-clamp trajectories of the N3A motif in R241A (left) and wild type (right). e, Force-dependent lifetimes of the N3A motif in the folded (triangles) and unfolded (circles) states for R241A (red) and wild type (blue). f, The mutation R241A (left) hinders interactions established with the CNB-B domain seen in wild type (right). 
a

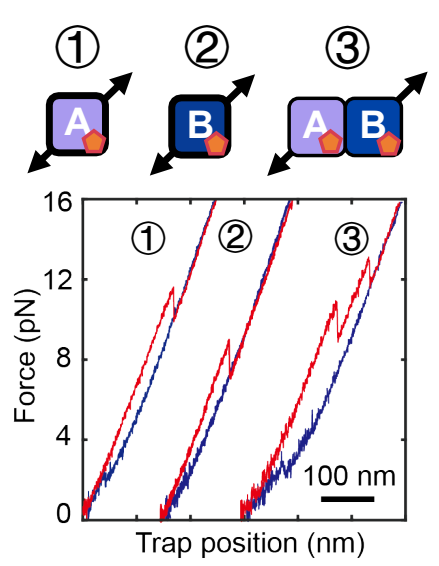

b
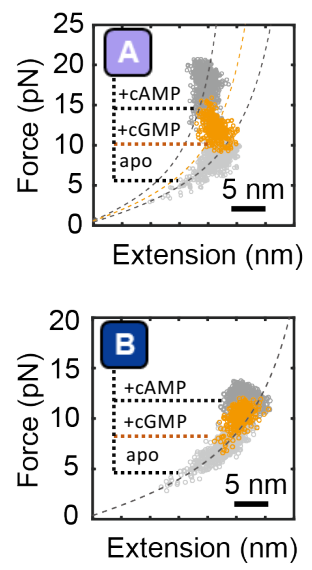
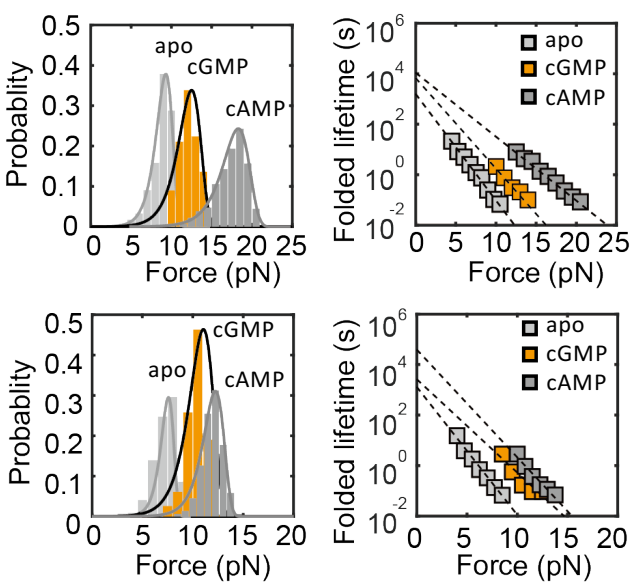

C
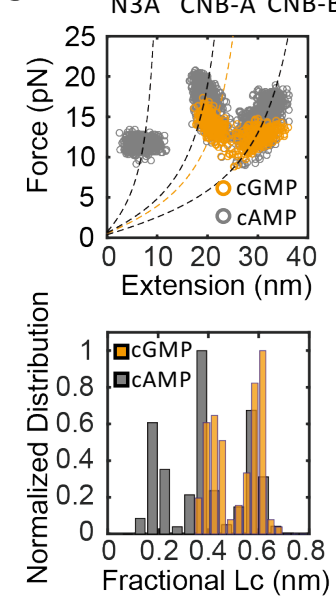

Figure 7 | Perturbation of allosteric networks in PKA by cGMP. a, Force-extension curves of cGMP-bound protein constructs. b, WLC analysis of changes in extension upon unfolding vs. force (left), unfolding force probability distributions (center) and force-dependent folded state lifetimes (right) for the isolated CNB-A (top) and CNB-B (bottom) domains in the apo (light gray), cGMP-bound (orange), and cAMP-bound (dark gray) states. Solid lines in center panels are the unfolding force distribution reconstructed from force-dependent lifetimes. c, WLC analysis of changes in extension upon unfolding vs. force (top) and fractional contour length (bottom) of the regulatory subunit bound to cGMP (orange) and cAMP (gray). Dashed lines are the WLC curves for the N3A motif and the two CNB domains with cAMP (gray) and cGMP (orange). 


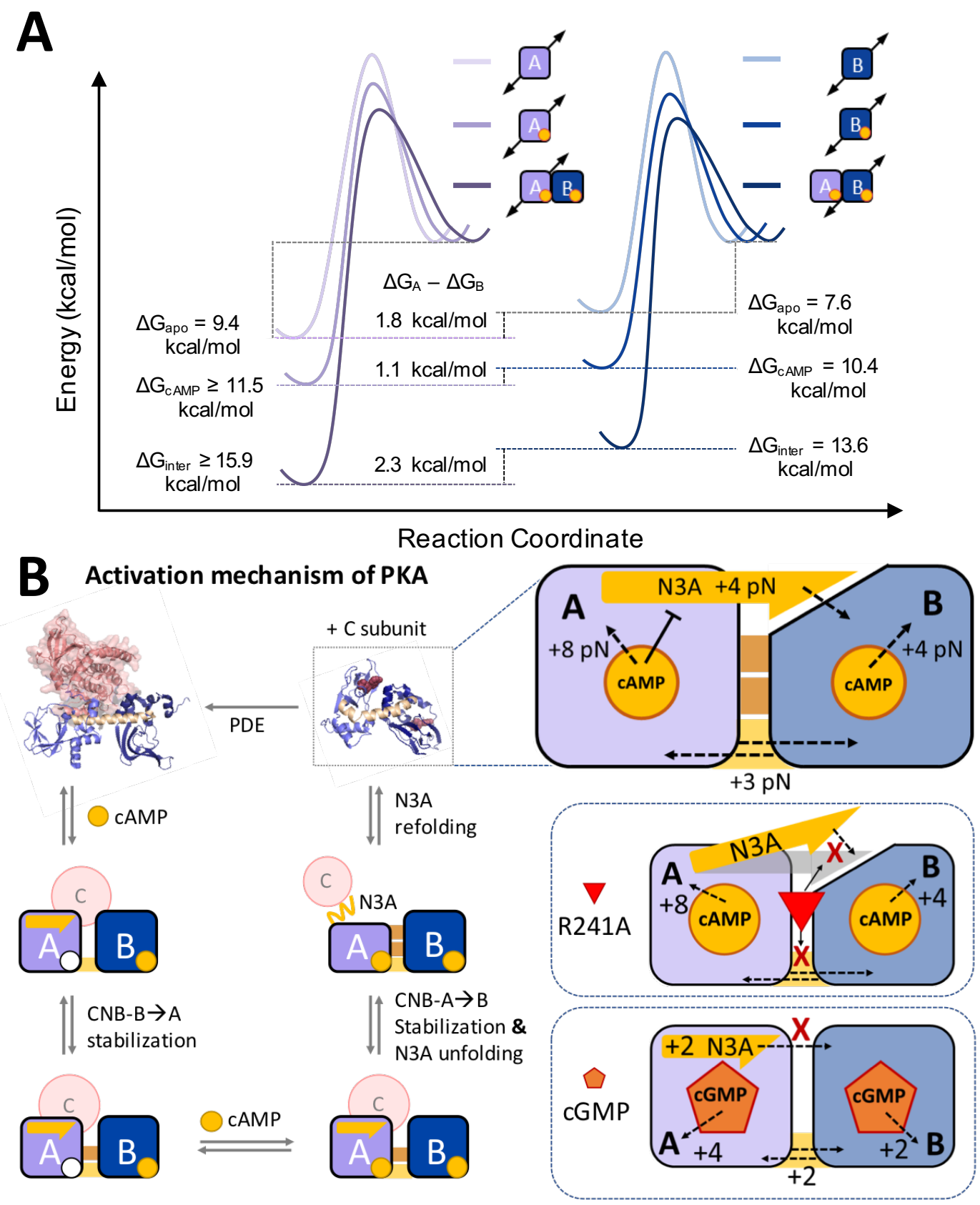

Figure 8 I Activation of PKA through selective stabilization of CNB domains a, Unique response of the energy landscape of the CNB domains due to cAMP binding and inter-domain contacts. The height of the energy barriers reflect the folded and unfolded state lifetimes of the CNB domains in the different configurations. b, Left: Activation mechanism of PKA showing intermediate states identified in this study. Right-top: Interaction network initiated by cAMP binding involves stabilizing (dashed black arrows) and destabilizing (flat black arrow) effects. Right-bottom: Interactions routes disrupted by R241A and cGMP. 
a

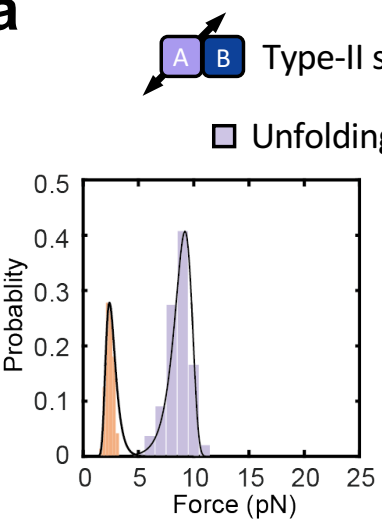

C
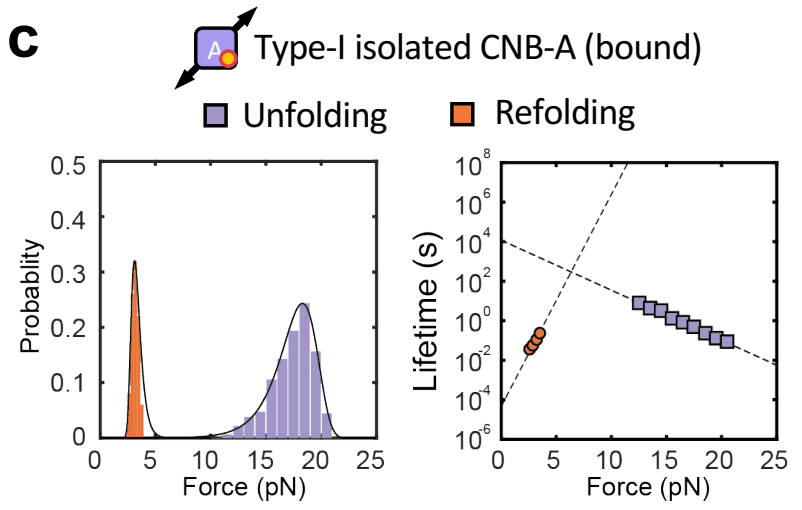

e
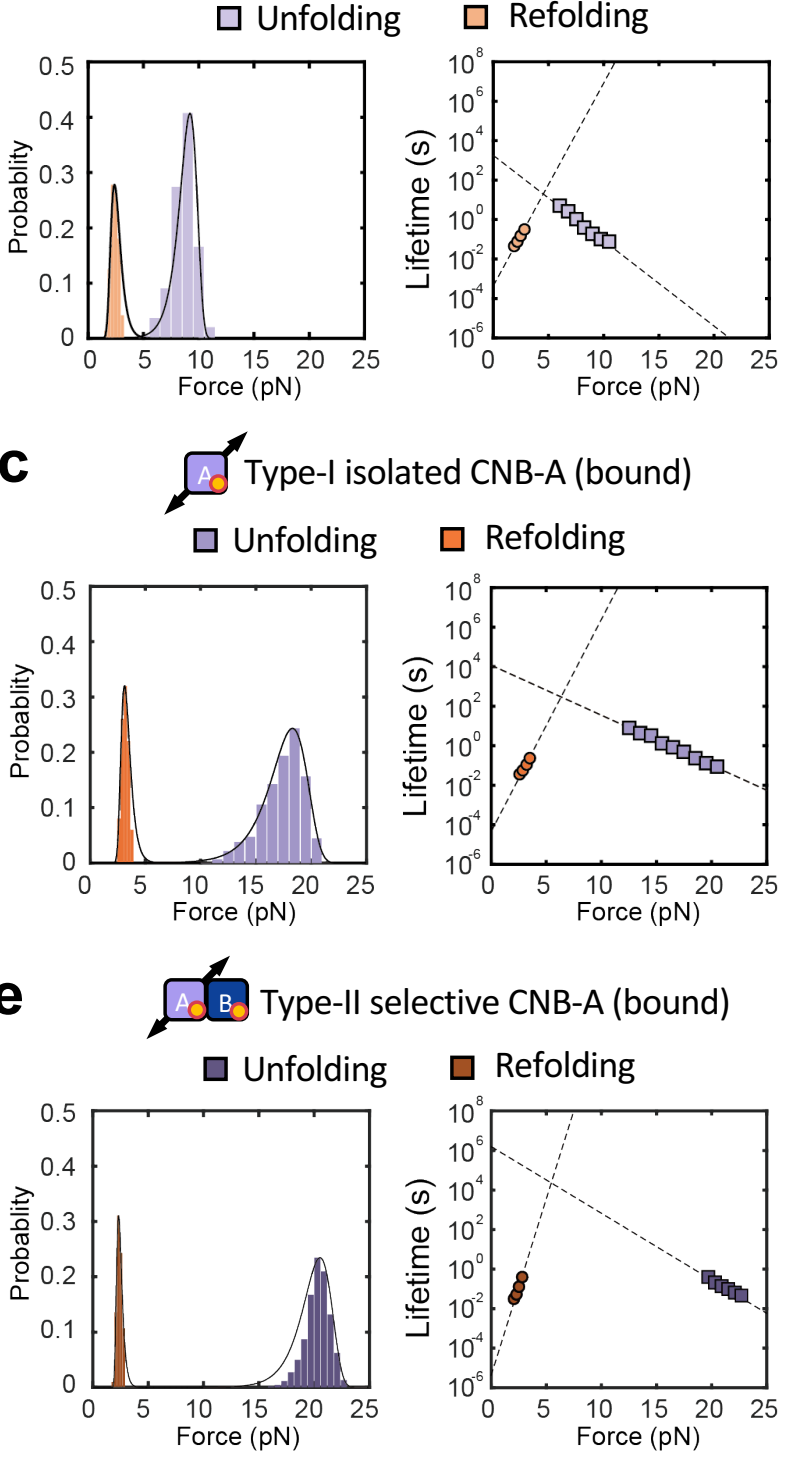

b
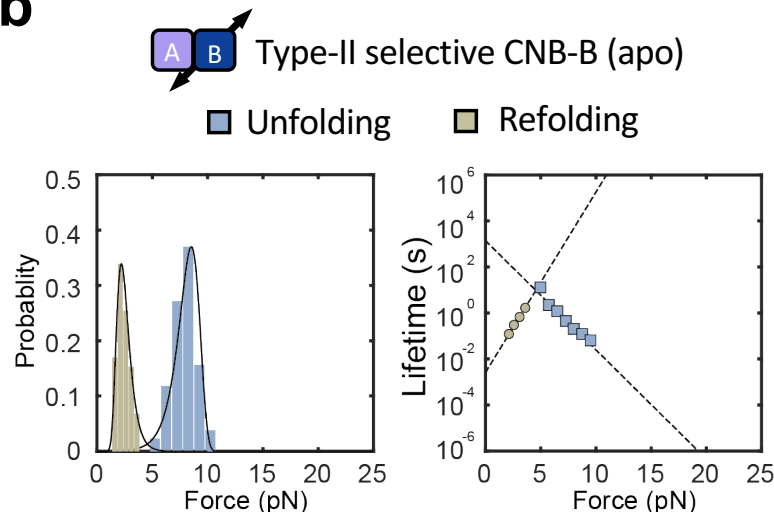

d

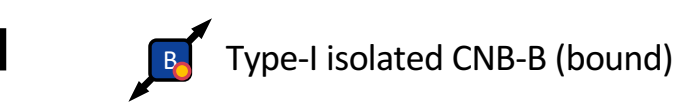

$\square$ Unfolding
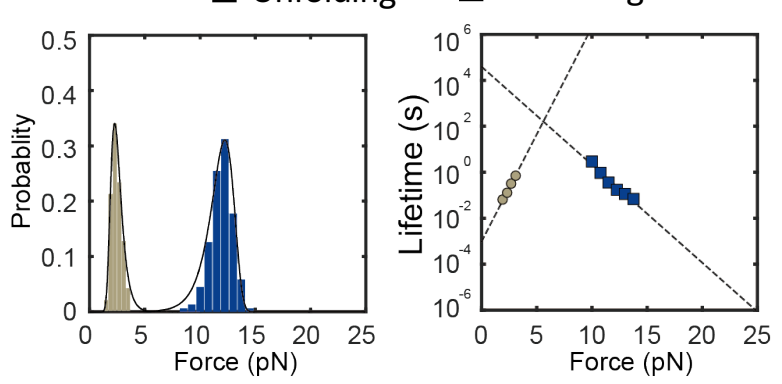

$f$ Type-II selective CNB-B (bound) unfolding

Refolding

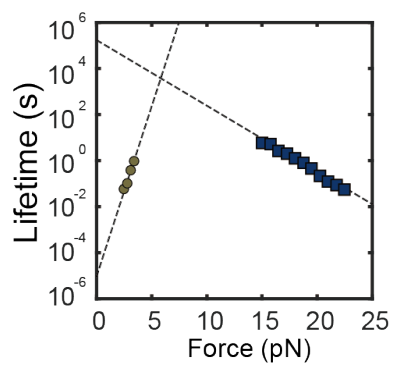

Supplementary Figure 1 | Unfolding and refolding force probability distributions and associated force-dependent folded and unfolded state lifetime with and without cAMP. a, CNB-A domain in type-II construct in apo state $\mathbf{b}, \mathrm{CNB}-\mathrm{B}$ domain in type-II construct in apo state. $\mathbf{c}$, CNB-A domain in type-I construct in cAMP-bound state. d, CNB-B domain in type-I construct in cAMP-bound state. e, CNB-A domain in type-II construct in cAMP-bound state. f, CNB-B domain in type-II construct in cAMP-bound state. 

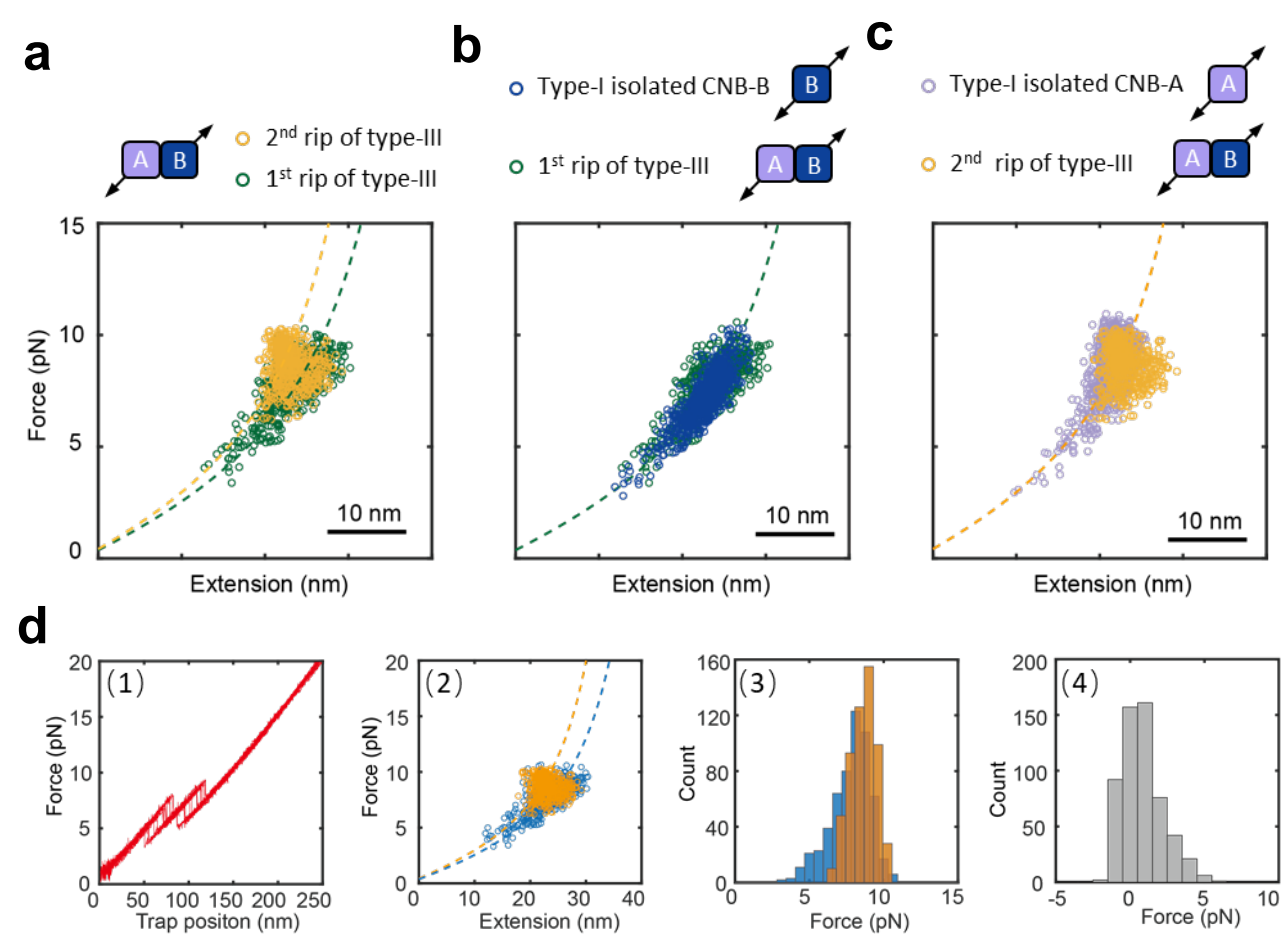

e
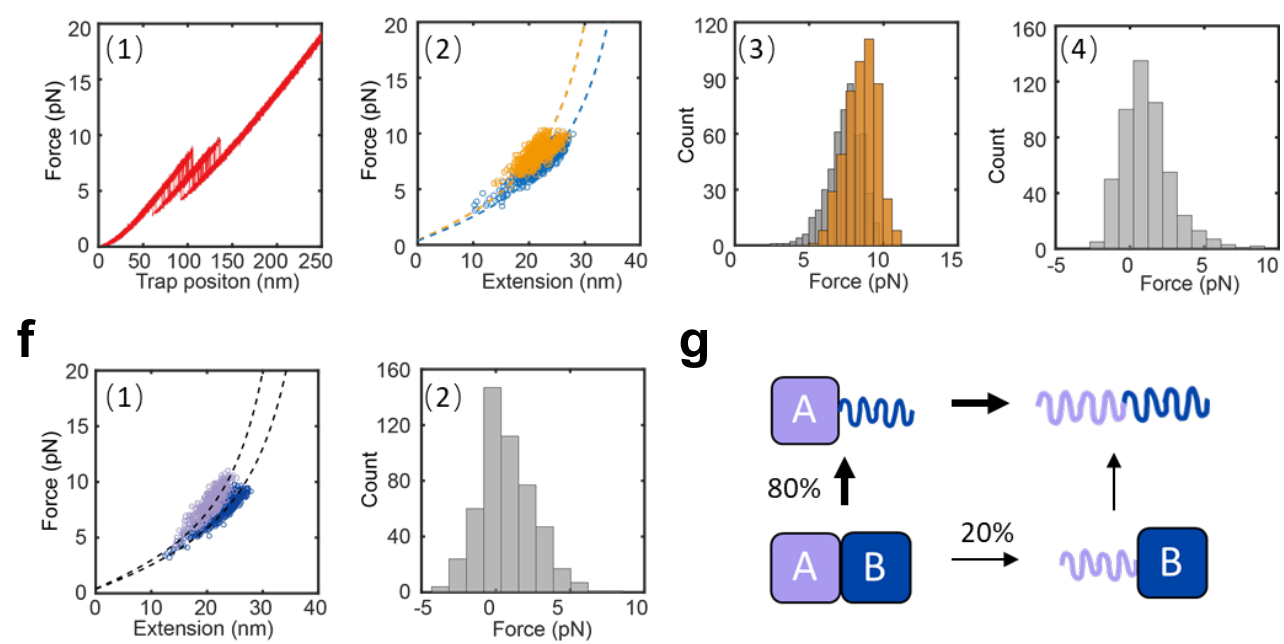

Supplementary Figure 2 । WLC analysis and Monte-Carlo simulations of type-III (S110C/S376C) and type-I constructs. a, Change in extension upon unfolding vs. force of the two rips observed in the type-III construct. The dashed lines are the experimentally determined WLC curves for the isolated CNB-A domain (orange, $\triangle \mathrm{Lc}=43 \mathrm{~nm}$ ) and the CNB$\mathrm{B}$ domain (green, $\Delta \mathrm{Lc}=50 \mathrm{~nm}$ ). $\mathbf{b}$, The $1^{\text {st }}$ unfolding rip in the type-III construct is indistinguishable from those obtained using a type-I CNB-B domain. The dashed line is the experimentally determined WLC curve for the CNB-B domain (green, $\Delta \mathrm{Lc}=50 \mathrm{~nm})$. c, The $2^{\text {nd }}$ rip in the type-III construct is indistinguishable from those obtained using the type-I CNB-A domain. The dashed line is the experimentally determined WLC curve for the CNB-A domain (orange, $\Delta \mathrm{Lc}=43 \mathrm{~nm}$ ). $\mathbf{d}$, Experimental data $(\mathrm{N}=559)$. (1) Representative force-extension curve obtained with optical tweezers using the type-III construct. (2) WLC analysis of changes in extension upon unfolding vs. force for the $1^{\text {st }}$ (blue) and $2^{\text {nd }}$ rips (orange). The WLC curves were generated using a $\Delta \mathrm{Lc}$ of $43 \mathrm{~nm}$ (yellow) and $50 \mathrm{~nm}$ (blue) corresponding to the CNB-A and CNB-B domains, respectively (SI Section 7). (3) Unfolding force histogram for the $1^{\text {st }}$ and $2^{\text {nd }}$ rips. (4) Unfolding force difference: $2^{\text {nd }}$ rip minus $1^{\text {st }}$ rip. e, Monte-Carlo simulation (N=559). (1) Simulated force-extension curves of the type-III construct were obtained using the kinetic parameters from the individual CNB domains (SI Table 1 and SI Section 8). (2) WLC analysis of the $1^{\text {st }}$ (blue) and $2^{\text {nd }}$ rips (orange) extracted from the simulated force-extension curves. The WLC curves were the same as in 2A. (3) Simulated unfolding force histogram for the $1^{\text {st }}$ and $2^{\text {nd }}$ rips. (4) Simulated unfolding force difference: $2^{\text {nd }}$ rip minus $1^{\text {st }}$ rip. f, (1) The Monte-Carlo simulation revealed the identity of the each CNB domain in the plot of changes in extension vs. force. Data for the CNB-B domain is in blue and corresponds to the $1^{\text {st }}$ rip in $80 \%$ of all simulated trajectories. Data for the CNB-A domain is in purple. The WLC curves were the same as in 2A. (2) Simulated unfolding force difference between CNB domains: CNB-A minus CNB-B g, Cartoon representing the unfolding pathway of the typeIII construct reconstructed from the Monte-Carlo simulation. ( For detailed analysis, refer to SI Section 2-3) 
a
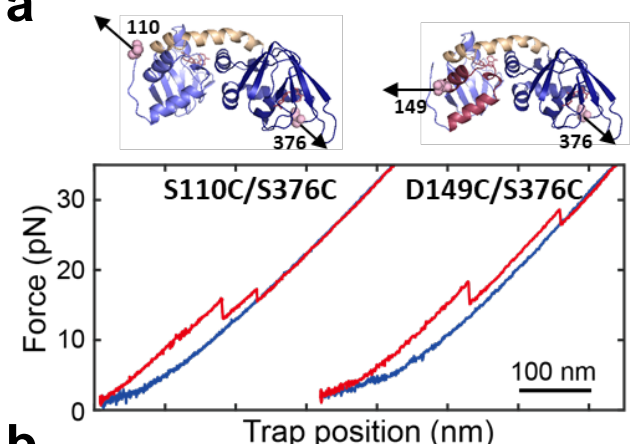

b
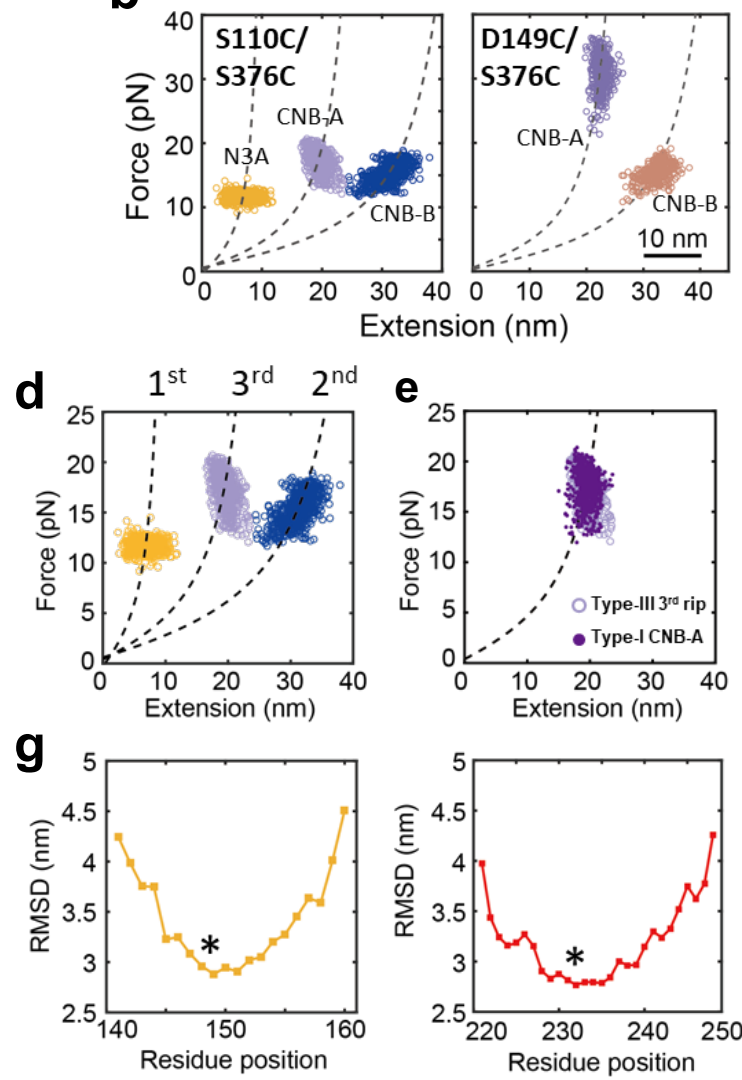

C
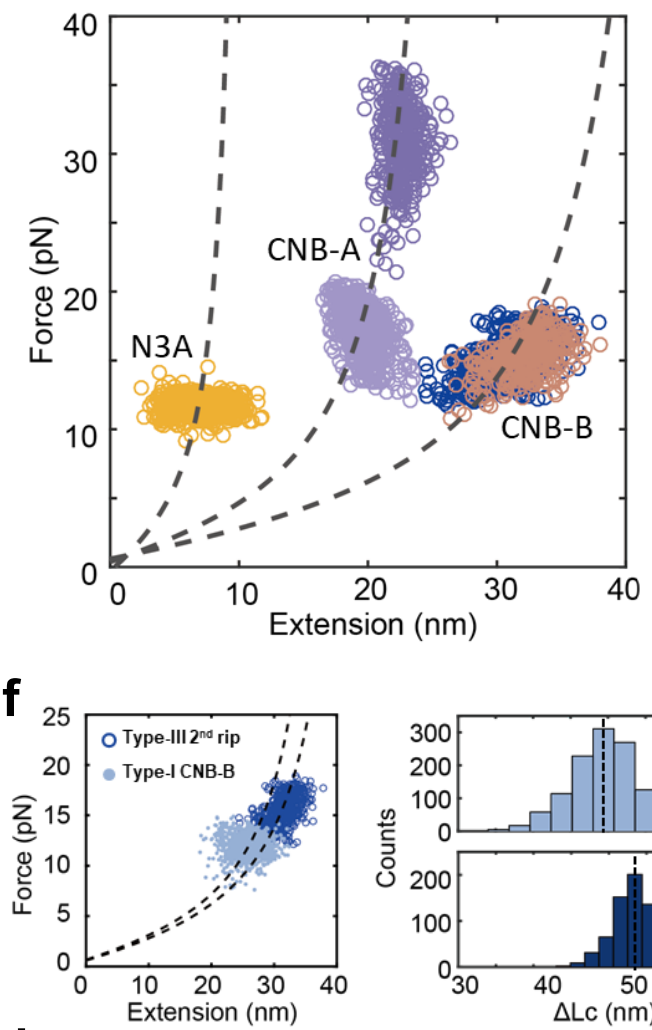

h
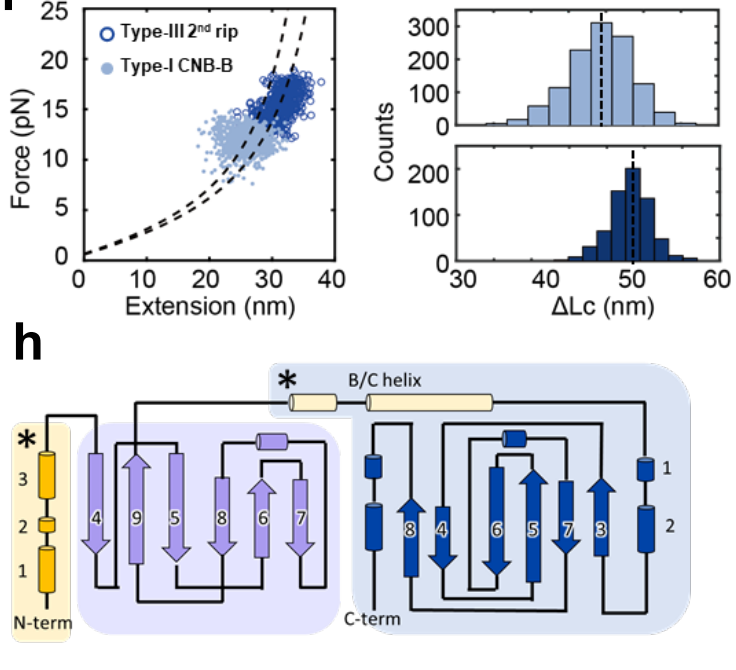

Supplementary Figure 3 । Identification of the N3A motif and assignment of unfolding intermediate for type-III construct in cAMP-bound state. a, Force-extension curve of type-III constructs with DNA handles at residue positions S110C/S376C (left) and D149C/S376C (right). Curves were obtained with cAMP. b, WLC analysis of changes in extension upon unfolding vs. force obtained for S110C/S376C (left) and D149C/S376C (right). The WLC curves (dashed lines) were obtained using a $\Delta \mathrm{Lc}$ of $13 \mathrm{~nm}$ (for the N3A motif), $31 \mathrm{~nm}$ (for the CNB-A domain) and $50 \mathrm{~nm}$ (for the CNB-B domain). The D149C/S376C does not show a transition corresponding to the N3A motif. c, Combined plot of changes in extension upon unfolding vs. force obtained for S110C/S376C and D149C/S376C. d, WLC plot of type-III construct (S110C/S376C) with cAMP. The WLC curves (dashed lines) were obtained using a $\Delta$ Lc of $13 \mathrm{~nm}$ (for the N3A motif, yellow), $31 \mathrm{~nm}$ (for the CNB-A domain, purple) and $50 \mathrm{~nm}$ (for the CNB-B domain, blue). e, WLC analysis (using $\Delta \mathrm{Lc}=$ $31 \mathrm{~nm}$ ) of the changes in extension upon unfolding for the CNB-A domain in the regulatory subunit (type-III construct) overlaid with the data obtained with the isolated CNB-A domain (type-I construct). f, WLC analysis (using $\Delta \mathrm{Lc}=47$ and $50 \mathrm{~nm}$ ) of the changes in extension upon unfolding for the CNB-B domain in the regulatory subunit (type-III construct, dark blue symbols) overlaid with the data obtained with the isolated CNB-B domain (type-I construct, light blue symbols). The CNB-B domain in the regulatory subunit has a longer $\Delta \mathrm{Lc}$ (right panels) likely due to the complete folding of the $\mathrm{B} / \mathrm{C}$ helix. g, Mapping the measured $\Delta \mathrm{Lc}$ for each unfolding rip to the corresponding structural elements of the regulatory subunit. Root-mean-square-deviation (RMSD) of the measured extension changes from the predicted WLC extension change as a function of the residue position for the $1^{\text {st }}$ rip (yellow) and $2^{\text {nd }}$ rip (red). The residue that gives the lowest RMSD is labelled with a star. These residues correspond to 149 ( $1^{\text {st }}$ rip) and 233 ( $2^{\text {nd }}$ rip). $\mathbf{h}$, Topology of the regulatory subunit showing the identified structural elements in each unfolding rip. The $1^{\text {st }}$ rip corresponds to unfolding of the N3A motif (residues 120-149), the $2^{\text {nd }}$ rip corresponds to the CNB-B domain with the BC helix (residues 233-376), and the $3^{\text {rd }}$ rip corresponds to the CNB-A domain minus the N3A motif (residues 150-233). 

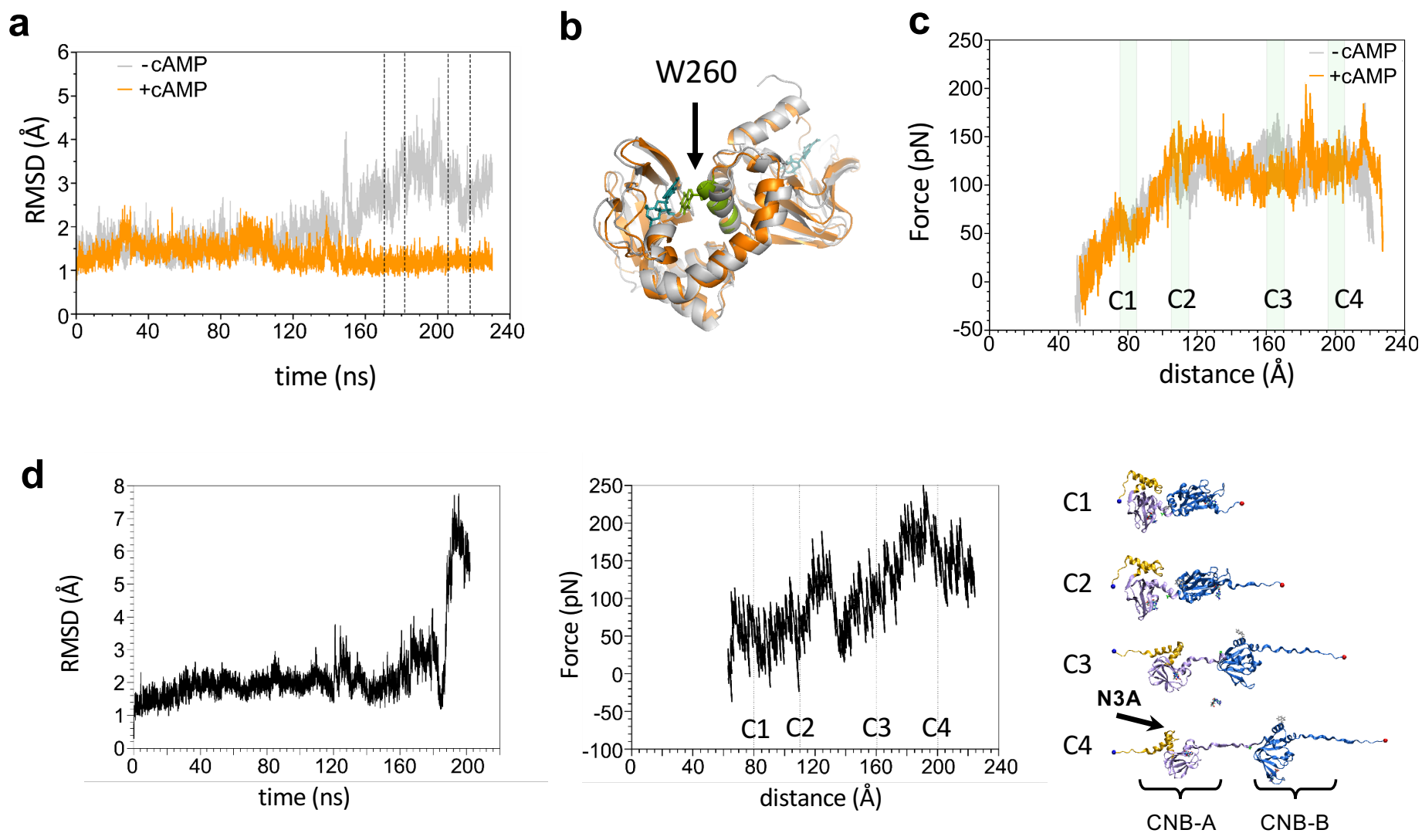

Supplementary Figure 4 I Steered molecular dynamic (SMD) simulations. a, Time series of the RMSD from the crystal structure for the PKA regulatory subunit with (orange) and without cAMP (gray). Vertical dotted lines indicate the frames used as starting points for SMD simulations. b, Representative structure of the most populated clusters in the last $50 \mathrm{~ns}$ of MD simulations with (orange) and without cAMP (gray); highlighted W260 interacting with cAMP docked in the binding site of the CNB-A domain. In the apo state, the lack of the interaction between cAMP and W260 and the absence of cAMP in the CNB-B binding site promote conformational rearrangement of the $\alpha \mathrm{A}: \mathrm{B}$ helix (green) causing a reciprocal orientation of the domains. c. Force-extension profiles for all the SMD simulations with (orange) and without cAMP (gray). Cluster analysis over selected structures (light green shaded areas) were used to characterize the most probable conformations along the trajectories. Yellow: N3A motif; Purple: CNB-A; Dark blue: CNB-B domain. d, RMSD time series (left) for MD, force-extension profile for SMD simulations (middle) and selected conformations (gray dashed lines) along the trajectories (right) for mutant R241A with cAMP along SMD. 

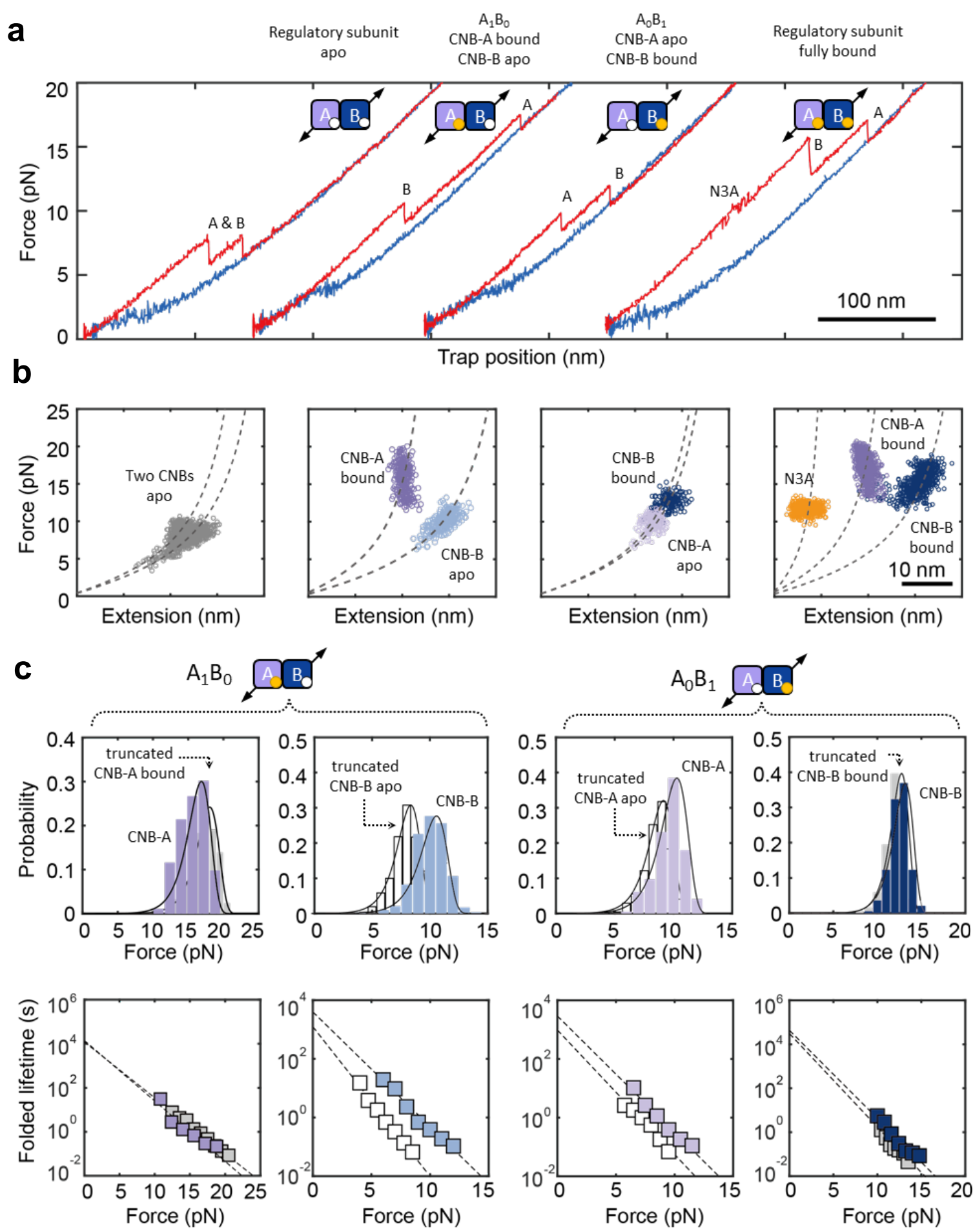

\section{Supplementary Figure 5 | Analysis of intermediate liganded states in cAMP titration of type-III} construct. a, Representative force-extension curves of four cAMP-bound states using the type-III construct. b, Corresponding WLC analysis of changes in extension upon unfolding vs. force. c, Unfolding force probability distributions and force-dependent folded state lifetimes of each CNB domain in two intermediate liganded states. The corresponding truncations in the ligand-free (white) and ligand-bound (gray) state were superimposed for comparison. 

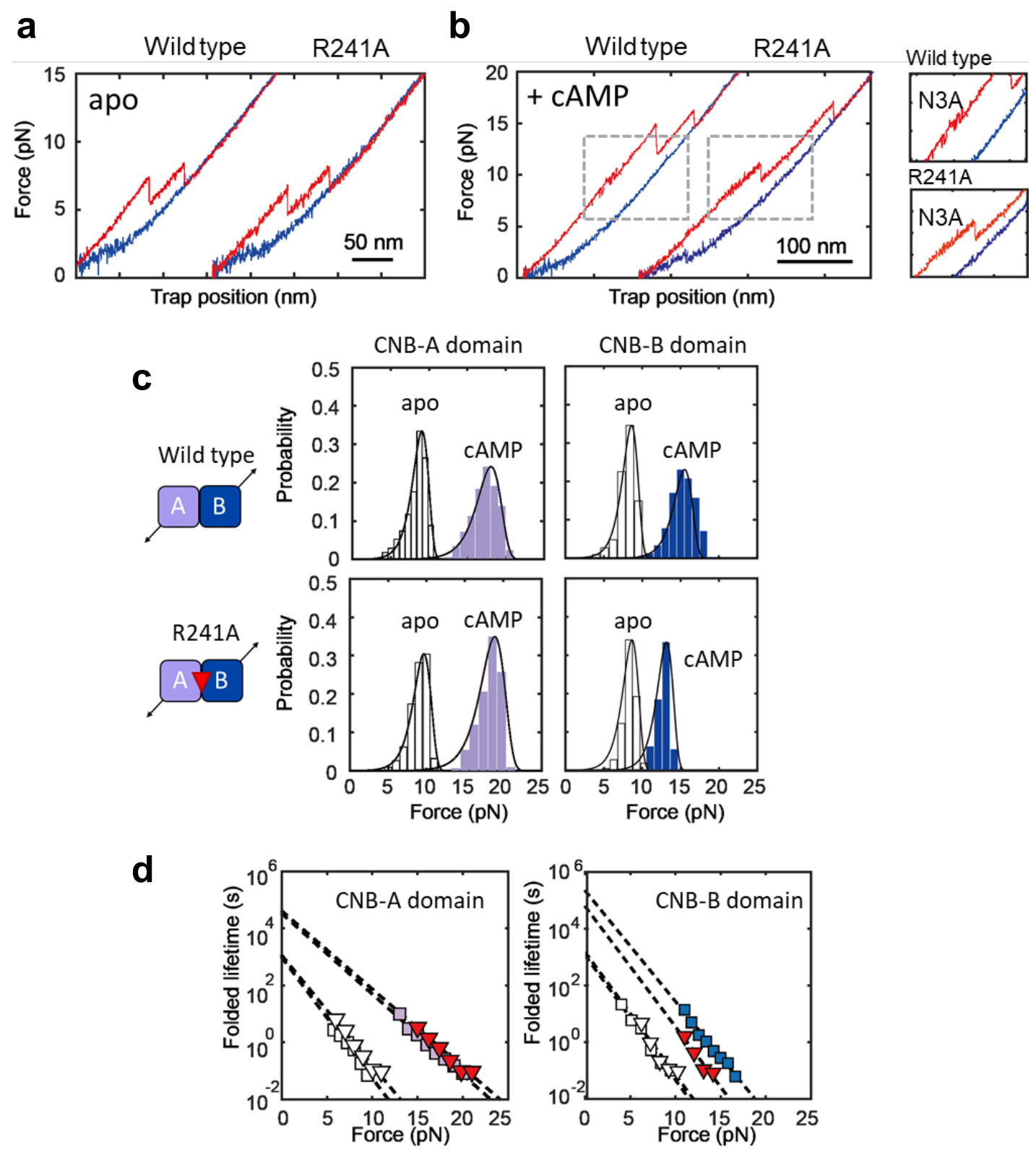

\section{Supplementary Figure 6 I Comparison of type-III (S110C/S376C) wild type and R241A mutant. a,} Force-extension curves of type-III constructs (S110C/S376C) for wild type and the R241A mutant regulatory subunits in apo state. b, Force-extension curves of type-III constructs (S110C/S376C) for wild type and the R241A mutant regulatory subunits obtained with cAMP (left). Zoomed-in trajectories showing the first reversible transition corresponding to the N3A motif in wild type and R241A (right). c, Unfolding force probability distributions for each CNB domain corresponding to wild type and R241A. The unfilled bar represents the apo state while filled bars represent cAMPbound data. d, Force-dependent folded state lifetimes for each CNB domain in the apo state (empty symbols) and cAMP-bound conformation (colored symbols) for wild type (squares) and R241A (triangles). 
a

CNB-A in type-III S110C/S376C apo

CNB-A in type-III S110C/S376C + cGMP

CNB-A in type-III S110C/S376C + cAMP
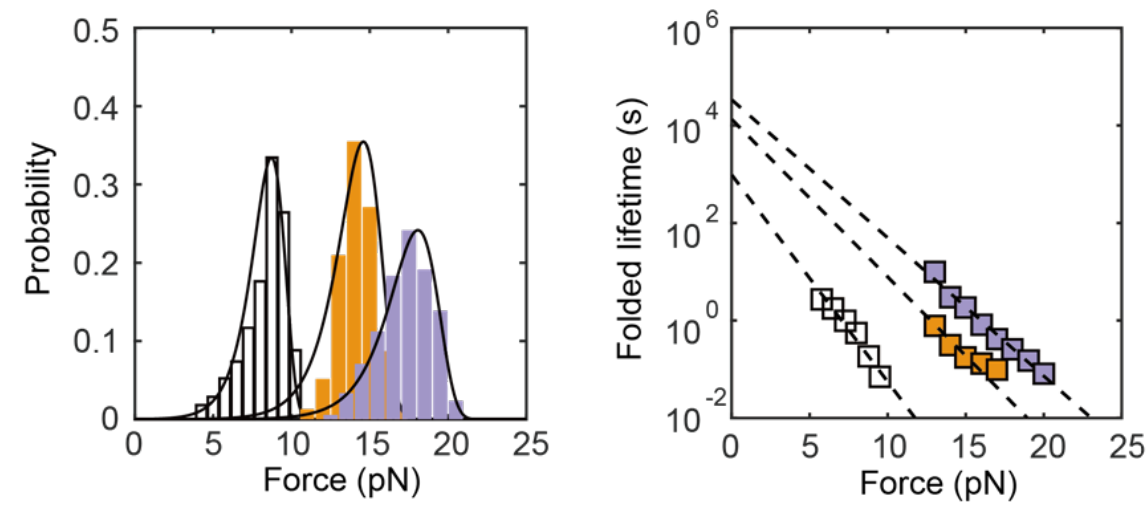

b

$\square$ CNB-B in type-III S110C/S376C apo

$\square$ CNB-B in type-III S110C/S376C + cGMP

$\square$ CNB-B in type-III S110C/S376C + cAMP
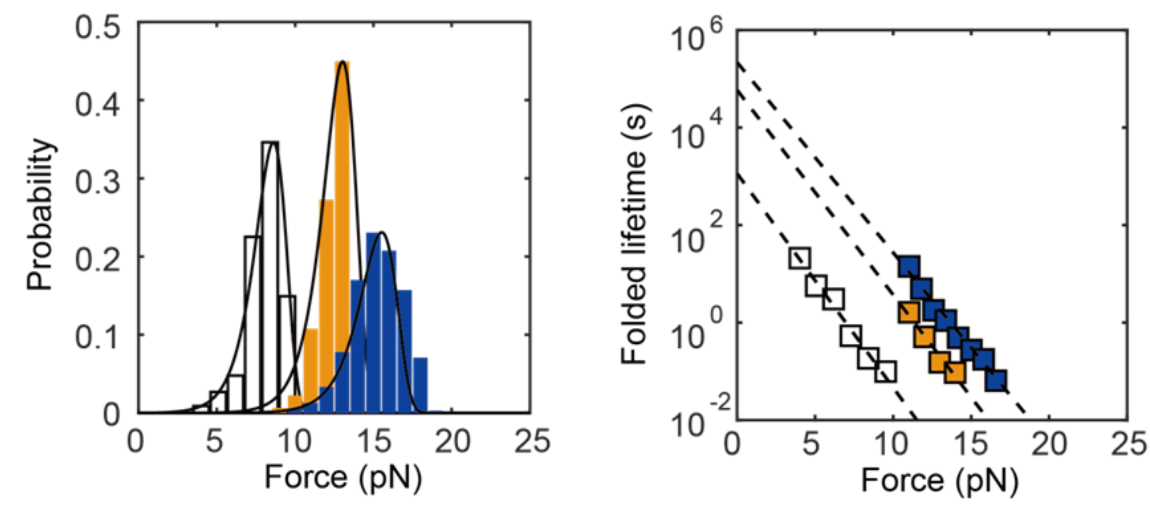

Supplementary Figure 7 | Comparison of unfolding force probability distributions (left) and corresponding force-dependent folded state lifetimes (right) for the type-III construct in apo, cGMP-, and cAMP-bound states. a, CNB-A domain and b, CNB-B in the regulatory subunit (typeIII construct, S110C/S376C) in the apo state (empty symbols) or bound to cGMP (orange) or cAMP (purple and blue). 